\title{
GKNet: grasp keypoint network for grasp candidates detection
}

\author{
Ruinian Xu, Fu-Jen Chu and Patricio A. Vela ${ }^{1}$
}

\begin{abstract}
Contemporary grasp detection approaches employ deep learning to achieve robustness to sensor and object model uncertainty. The two dominant approaches design either grasp-quality scoring or anchor-based grasp recognition networks. This paper presents a different approach to grasp detection by treating it as keypoint detection in imagespace. The deep network detects each grasp candidate as a pair of keypoints, convertible to the grasp representation $g=\{x, y, w, \theta\}^{T}$, rather than a triplet or quartet of corner points. Decreasing the detection difficulty by grouping keypoints into pairs boosts performance. To promote capturing dependencies between keypoints, a non-local module is incorporated into the network design. A final filtering strategy based on discrete and continuous orientation prediction removes false correspondences and further improves grasp detection performance. GKNet, the approach presented here, achieves a good balance between accuracy and speed on the Cornell and the abridged Jacquard datasets (96.9\% and $98.39 \%$ at 41.67 and $23.26 \mathrm{fps})$. Follow-up experiments on a manipulator evaluate GKNet using 4 types of grasping experiments reflecting different nuisance sources: static grasping, dynamic grasping, grasping at varied camera angles, and bin picking. GKNet outperforms reference baselines in static and dynamic grasping experiments while showing robustness to varied camera viewpoints and moderate clutter. The results confirm the hypothesis that grasp keypoints are an effective output representation for deep grasp networks that provide robustness to expected nuisance factors.
\end{abstract}

\section{Keywords}

Grasping, Recognition, Learning and Adaptive Systems

\section{Introduction}

Robotic manipulation in unstructured and dynamic environments is a crucial task when deploying robots for daily living and industrial activities. Grasp detection is an essential subproblem of robotic manipulation since knowing where and how to grasp objects is a starting point for manipulation. Real-world use of robot manipulators demands accurate, robust, and efficient or real-time operation. Contemporary solutions aim to exhibit all of these characteristics, however there usually exists a trade-off between them. Deep learning based approaches for grasp detection have best balanced these needs to achieve state-of-the-art performance. Research for grasping roughly splits into 4 Degree-ofFreedom (DoF) and 6 DoF methods, with 4 DoF methods exhibiting a more favorable balance. This paper focuses on 4 DoF grasp recognition such that the output grasp representation is specified in image coordinates, and converted to a 6 DoF equivalent through the assumption of top-down grasping. Image-based, deep learning approaches tend to be convolutional neural network $(\mathrm{CNN})$ approaches (Redmon and Angelova 2015; Kumra and Kanan 2017; Guo et al. 2017; Zhou et al. 2018; Chu et al. 2018; Asif et al. 2019). The prevailing neural network approaches divide into one-stage and two-stage grasp detectors, and grasp quality scoring networks. One-stage grasp detectors generate grasp predictions with a single deep network, whereas two-stage grasp detectors generate candidate detection regions with one deep network and then evaluate the feature vectors of the candidate regions using a second network to generate grasp hypotheses. Compared to one-stage grasp detectors, twostage grasp detectors typically achieve better performance by

predicting potential grasp regions. However the incorporation of a grasp region proposal network increases inference latency. One-stage grasp detectors commonly run at realtime speed but with reduced performance.

Contemporary computer vision research into reducing the performance gap between one-stage and two-stage detection methods investigates alternative detection representations within the processing pipeline. One architecture commonly used is anchor boxes (Ren et al. 2015); a series of boxes whose size and aspect ratio lie within a specified parametric domain. Much like for region proposals, a major drawback of the anchor boxes is the introduction of additional hyperparameters, which require manual specification to optimize performance. These hyperparameters specify the total number of anchor boxes and their geometry (such as sizes and aspect ratios). Hyperparameter choices impact detection outcomes. Another achitecture is that of keypoints (Law and Deng 2018; Duan et al. 2019); it represents bounding boxes as a set of keypoints. Instead of directly regressing parameters for bounding boxes, keypoint detection approaches perform pixel-level segmentation and yield a set of keypoint heatmaps. These keypoint maps are then grouped and post-processed to form the final detections.

\footnotetext{
${ }^{1}$ Intelligent Vision and Automation Laboratory (IVALab), School of Electrical and Computer Engineering, Institute for Robotics and Intelligent Machines; Georgia Institute of Technology, GA, USA.
}

\section{Corresponding author:}

Ruinian Xu, Intelligent Vision and Automation Laboratory (IVALab), Georgia Institute of Technology, North Ave NW, Atlanta, GA 30332.

Email: rnx94@gatech.edu 


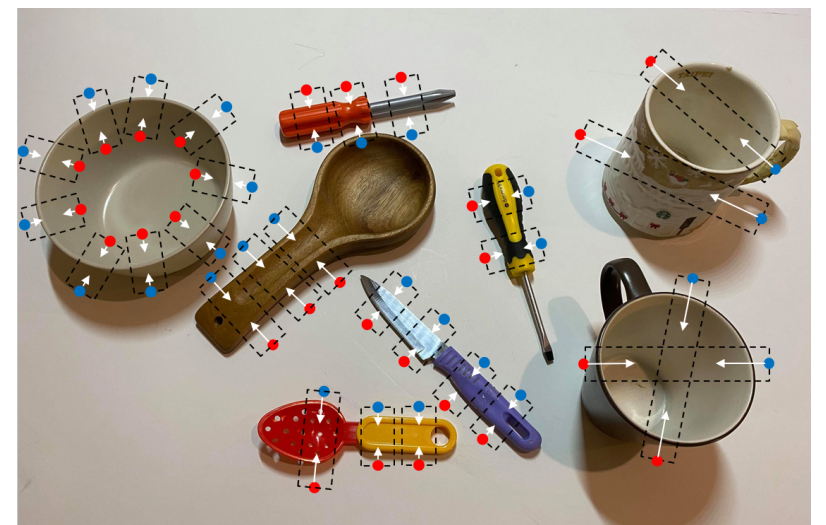

Figure 1. Illustration of grasp candidate detection with proposed grasp representation and method. The red points are right keypoints. The blue points are left keypoints. The black boxes correspond to associated grasp bounding boxes for keypoint pairs. For illustration of the grasp, the white arrows indicate the closure directions for parallel-jaw grippers.

Keypoint detection methods are one-stage approaches that achieve competitive performance with two-stage approaches.

This paper describes a one-stage grasp detector that solves grasp detection as a keypoint detection problem. The most general image-based grasp representation, $g=$ $(x, y, \theta, w, h)^{T}$, describes an oriented bounding box. Though applicable to a variety of grippers, it is best suited to parallel-plate/jaw gripper types. For keypoint architectures, specifying an oriented bounding box would involve either three or four corner points, whose geometry must meet orthogonality constraints to properly define a box shape. Instead of a fully-specified grasp, the proposed approach represents oriented bounding boxes as grasp keypoint pairs: the left-middle and right-middle points of the grasp bounding box (see Fig. 1). Doing so reduces the necessary geometric constraints and also the difficulty of grouping predicted keypoints to arrive at grasp candidates, as only pair-wise keypoint matches are needed (versus triple or quadruple correspondences). The reduction speeds up and simplifies the grouping process, and provides for additional means to internally certify correctness of the grasp hypothesis. Furthermore, due to the difficulty of encoding geometric constraints between grasp keypoints into the training objective function, removing redundant components benefits keypoint detection methods, since even one bad predicted keypoint can significantly change the bounding box geometry. A byproduct of the reduced representation is the loss of the bounding box length coordinate, $h$. The loss is minimal since most (parallel-plate) grippers have constant thickness fingertips. A variable $h$ is rarely implemented.

The benefits of a left-middle and right-middle keypoint representation translates to neural network processing architecture. The key-point representation requires less information to synthesize a valid grasp candidate. Another consequence is that it permits simpler grasp validation strategies and the incorporation of strategies to couple the left and right key-point detection processes within the feature space processing. The net outcome is to better balance trade-offs in performance and processing speed. The proposed deep network, called GKNet, is a one-stage neural network that maps RGB-D input images to rankordered $\{x, y, \theta, w\}^{T}$ grasp candidates. The GKNet pipeline is depicted in Fig. 2 and detailed in Section 4. Internally, the network generates two grasp keypoint heatmaps, left and right, along with additional maps to improve keypoint pairing performance and localization. These maps are the embedding map, offset map, and center keypoint heatmap. Post-processing of the network output groups left and right keypoints into pairs representing grasp hypotheses based on a series of validation and filtering conditions using the embedding maps and the center keypoint heatmap.

To quantify the balance between performance and processing speed, GKNet is compared to baseline algorithms on the purely visual problem of grasp detection (Section 6 ). Relative to the baselines, it achieves good trade-off between accuracy and speed on the Cornell and Jacquard Datasets (Lab 2013; Depierre et al. 2018). It lies at the extremal corner of the convex hull of all methods in the accuracy/speed plane. GKNet achieves competitive performance with two-stage approaches. To quantify performance when deployed on an actual manipulator, GKNet is integrated into a manipulation pipeline and tested on four physical grasping experiments: (a) static grasping, (b) dynamic grasping, (c) grasping at varied camera viewpoints and (d) bin picking. These tests, described in Section 7, evaluate the system with respect to accuracy, speed, and robustness. Reported experiment outcomes in Section 8 include comparative discussions and analysis. Performance outcomes across the suite of benchmarks for static grasping, which include some existing one-stage and two-stage grasp detectors, demonstrate that GKNet is capable of predicting more accurate grasping candidates. Dynamic grasping experiment results show that GKNet provides reliable grasp predictions in real-time. Grasping at varied camera poses and bin picking experiments illustrate the robustness of GKNet under different camera views and in cluttered environments scenarios, which are not included in the training dataset. The consistent performance of GKNet across these experiments provides evidence in favor of keypoint representations for grasp recognition.

\section{Related Work}

Research emphasis on robotic grasp detection has shifted over the past two decades from analytical methods to empirical methods. The review papers (Bicchi and Kumar 2000; Sahbani et al. 2012; Shimoga 1996) describe conventional analytical methods. They perform grasp candidate analysis based on prior knowledge, like object geometry or pose, grasp closure and force analytics, and/or physical models. The major drawback lies in the assumption that the object parameters informing the grasp analysis are known, which is hard to satisfy for general, real-world manipulation. Empirical methods, also known as learningbased or data-driven methods, perform grasp detection using functional models learned from available real or synthetic grasp results and their input signals (usually imagery). When given diverse inputs, empirical methods generalize to novel cases. This section reviews existing grasp detection approaches with an emphasis on the empirical methods.

Additionally, the focus will be on parallel-jaw grippers or their equivalents given that a large class of objects 


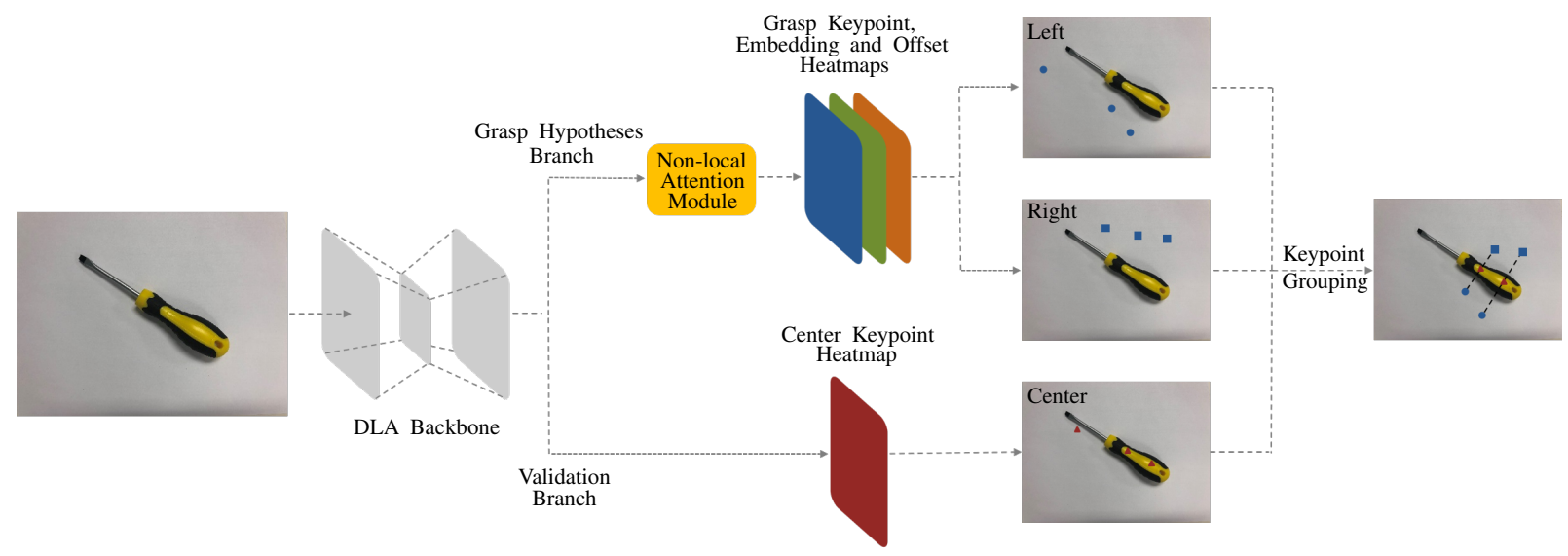

Figure 2. Architecture of GKNet. An hourglass-like backbone network is followed by two prediction branches, one for grasp keypoints and one for a center keypoint. A non-local attention module is inserted between backbone network and the prediction branch for grasp keypoints. The pair of grasp keypoint heatmaps with corresponding embeddings and offsets and the center keypoint heatmap are fed into keypoint grouping algorithm and yield the final grasp detections. Blue dots correspond to left grasp keypoints, blue rectangles correspond to right grasp keypoints and red triangles correspond to center keypoints.

can be grasped from two opposing contact regions. Where this class of grippers can fail is with non-smooth or asymmetric grasping surfaces. Multi-fingered grippers are capable of performing more complex grasp configurations (Lu et al. 2020; Kappler et al. 2015; Saxena et al. 2008b; Varley et al. 2015; Veres et al. 2017; Zhou and Hauser 2017). Alternatively, suction gripper platforms provide a different set of grasping affordances for grasping diverse categories of objects based on a single contact region (Eppner et al. 2016; Mahler et al. 2018; Shao et al. 2019) which has led to their wide deployment in warehouse or manufacturing settings. Their common use by industry is backed by recent research strategies that lead to suction grippers being favored over standard grippers due to their grasping performance advantage, when employing a bimanual suction and parallel-plate gripper robot configuration (Mahler et al. 2019; Zeng et al. 2018b; Yen-Chen et al. 2020; Fujita et al. 2020). Though highly capable when used in structured settings like warehouses or manufacturing, suction grasping limits the target objects to those with suction graspable surface geometry. This work aims to improve parallel-plate styled gripper grasp success and lower the performance gap for suction grippers while improving on use cases and scenarios where the latter are not used/available.

Continuing, early empirical methods for transferring prior knowledge of annotation grasps to novel objects employed manually designed feature representations. The idea of decomposing grasping into the identification of where and how to grasp (location and end-effector pose) and how well the location will grasp (quality) emerged as an important design template (Kamon et al. 1996). Such a decoupled learning process meant that location parameters were objectagnostic and could be applied to new objects. Likewise, the notion that learning might be best performed through demonstration instead of annotation has been a consistent thread of research activity. Demonstration can lead to direct mappings from the demonstrated human grasp postures to robot grasps (Ekvall and Kragic 2007), or indirect mappings that transform grasp contact points for objects in the human demonstration database to novel or known target objects (Hsiao and Lozano-Perez 2006).
Since then, advances in compute performance and machine learning, plus the generation of larger annotated datasets, contributed to increased study into supervised learning techniques. Employing learning approaches sourced from the computer vision community, methods for inferring grasp directly from visual data, RGB or RGB-D, quickly came to prominence (Saxena et al. 2008a; Le et al. 2010). Working in image space required defining grasps in terms of oriented rectangular grasp regions that were then mapped to an actual 3D grasp configuration and pre-grasp pose, then planned for execution (Jiang et al. 2011). Some methods chose instead to operate on the point clouds and surface representations (Pelossof et al. 2004; Rao et al. 2010). The top performing learning-based methods demonstrated robust grasping by manipulators with average success rates in the range of $85-90 \%$ for small sets of novel objects. Likewise, simulation methods emerged as a means to rapidly collect training data for well defined feature spaces (Pelossof et al. 2004). In some instances reinforcement learning over simple feature spaces was used to identify quality grasps (Piater 2002; Coelho et al. 2001). These early studies informed the conceptual framing of grasping, while later work has improved the outcomes through the use of deep learning methods with more robust inference properties. The remainder of this section reviews grasping based on deep neural network implementations.

\subsection{Deep Learning Methods}

The asserted benefit of deep learning is that the loss functions implicitly shape the internal feature space and free the designer from having to explicitly encode the input to feature space mapping. When combined with large datasets, presumably the internal feature space supports generalization to novel inputs or robustness to input variation. Deep networks have been used for grasp recognition, for grasp quality scoring, and within grasp policy reinforcement learning strategies. These are all reviewed below.

2.1.1 Grasp Quality Scoring. As an extension of their analytical predecessors, grasp quality scoring networks use deep networks to evaluate sampled grasp candidates and 
create a ranked ordering based on hypothesized grasp success scores. Instead of end-to-end mapping images to grasps or actions, these methods consider robotic grasping within a pipeline where earlier steps involve grasp sampling and later steps involve grasp planning algorithms. Treating grasp detection as a binary classification problem of graspable or not graspable is the most straightforward implementation (Gualtieri et al. 2016). This idea was extended in Pinto and Gupta (2016) with the inclusion of orientation dependent scoring implemented as an 18way classifier over discretized orientation classes. Since grasp samples may not densely nor sufficiently sample from the feasible grasp space, there is some advantage to using deep learning to first hypothesize candidate grasps (Lenz et al. 2015). In that case, a second network will then evaluate the detected candidates and provide a ranked ordering. When trained with binary labels from annotated grasp datasets, there will be an upper limit on performance since true grasp quality scores are not available. Based on the assumption that not all grasps have the same grasp success probability, extending binary labels to ordered labels in the annotations better informs the neural network learning process with regards to ranking grasp candidates (Johns et al. 2016). Once actual scores are available, the function output can be modified to perform continuous regression and avoid the quantization effects of classifier-based network designs (Mahler et al. 2017). Since doing so requires more annotations, Mahler et al. (2017) included the creation of Dex-Net 2.0, a dataset with 6.7 million points clouds each of which is tagged with grasps and their scores as generated by analytic grasp metrics. Continuing further, imitation learning employing simulated grasp scenarios can provide even richer training data for grasp scoring and annotation (Mahler and Goldberg 2017; Zeng et al. 2018a; Yen-Chen et al. 2020). Furthermore, the simulated scenarios can be custom tailored to specific manipulation tasks, such as bin picking (Mahler and Goldberg 2017). Besides learning a grasp quality function, some works employ other features for representing the graspability of grasp candidates. For example, the distance between the manipulator and the nearest viable grasp after executing the given action provides an indirect probability that the manipulator achieves the graspable configuration (Viereck et al. 2017).

2.1.2 2D Grasp Prediction. A sub-class of grasp detection or grasp recognition methods map the input images to a set of 2D oriented bounding boxes as the grasp representation, with the premise that follow-up processing of the grasp region will recover the actual 3D grasping approach to apply. The assumption of a top-down view with top-down grasping provides a direct conversion of the grasp representation to the $6 \mathrm{DoF}$ grasp to execute. These grasp recognition strategies decompose into onestage grasp detectors, two-stage grasp detectors, and lastly implementations that combine traditional image and pointcloud processing pipelines and manually engineered features with convolutional neural networks (Asif et al. 2017). The principle idea behind this last category is that some critical information is best computed and provided directly, as opposed to expecting the learning process to implicitly recover and encode the information within its convolutional structure and internal feature space. While annotation demands are reduced and performance is strong for the multipronged approach, contemporary research aims to simplify the processing pipeline while achieving high grasp success rates and high processing rates. Generally one-stage methods use a single neural network to directly regress single or multiple grasp candidates from an image (Redmon and Angelova 2015; Wang et al. 2016; Watson et al. 2017; Kumra and Kanan 2017). The most commonly used grasp representation is $(x, y, \theta, h, w) \in S E(2) \times \mathbb{R}^{2}$ (Redmon and Angelova 2015; Watson et al. 2017; Kumra and Kanan 2017). Some variations add extra or remove parameters such as the 4-D grasp representation consisting of center location, opening length and orientation angle (Wang et al. 2016). Although these approaches provided relatively high (around 90\%) grasp detection rate on the Cornell Dataset (Lab 2013), performance is lower than two-stage detectors.

An essential technique introduced to help one-stage grasp detectors break the performance ceiling was that of anchor boxes. A series of anchor boxes with different scales and aspect ratios predicts potential grasp candidates over the input image without the extra process of scanning the image with a sliding window technique. Applying the technique to grasping does reduce the performance gap somewhat, and can also incorporate tactile information to improve the grasp detection process (Guo et al. 2017). Extending to the case of oriented anchor boxes led to one-stage methods on par with two-stage methods, but with equivalent processing speed even though the one-stage method should be faster (Zhou et al. 2018). Abstracting the concept of anchor boxes to regions leads to networks that perform pixel-wise predictions over image regions. Predicting pixel-wise grasp hypotheses removes the need to define anchor box hyperparameters (Morrison et al. 2019; Satish et al. 2019). Both implementatios are grasp quality networks that also output grasp geometry parameters such as the rotation angle and opening (Morrison et al. 2019), or the 3-D position and planar orientation (Satish et al. 2019). While using anchor boxes improves the detection accuracy, they also introduce additional hyperparamters that requires manual specification to optimize performance.

Compared to one-stage approaches, two-stage grasp detectors require an extra region proposal network (RPN) for generating regions of interest (RoIs). A series of RoIs generated in the first stages are used to crop corresponding features over the entire image for identifying grasp candidates in the second stage. Evaluating all potential grasps, RPN helps locate coarse regions that may contain potential grasps. Furthermore, the two-stage mechanism achieves strong performance by first coarsely searching and then refining detections (Chu et al. 2018). Based on the fact that classification-, regression- and segmentation-based grasp detection methods have their own advantages and drawbacks, some researchers try to fuse features captured at different levels to improve on grasp recognition. The resulting network is a multi-branch deep neural network which predicts grasp candidates at global, regional, and pixel-levels Asif et al. (2019). Two-stage methods achieve good performance on grasp detection, but high inference latency may limit their translation to real-world applications. 
2.1.3 Reinforcement Learning. Reinforcement learning (RL) solves robotic grasping by learning end-to-end robot arm motion control policies from image inputs in a manner that couples the learning process with the experiential training data. RL is effective when ground truth annotations are difficult to come by. Predicted motions can be represented in joint-space (Levine et al. 2016a) or Cartesian task-space (Levine et al. 2016b). Research emphasis on this front tends to focus on manipulation or robust picking strategies targeted to specific tasks and working environments. For densely cluttered environments, grasp success rate is highly affected by object stacking configurations. Rearranging objects prior to grasping has value. Zeng et al. (2018a) added pushing to the action-space with the aim of learning synergies between pushing and grasping actions. Fusing grasp measurement signals, such as the pressure measured after performing a grasp, helps the robot determine re-grasp strategies. Calandra et al. (2018) combined tactile information with visual observation to learn an evaluation function that maps the current visuo-tactile observations and a candidate action to its probability of success. End-to-end learning approaches generate a locally optimal action response by end-to-end mapping observations to robotic motions but their application for real-world tasks remains uncertain given that a policy's robustness to configuration changes is not fully known. Better generalization capabilities for grasping have been shown using other batch-based learning methods. For example, more generalizable policy learning has also been achieved through the use of imitation learning (IL) strategies in the training process (Mahler and Goldberg 2017; Mahler et al. 2019; Satish et al. 2019). where IL has strong connections to RL.

2.1.4 3D Grasp Prediction. Contemporary grasp recognition methods with high success rates benefit from premises that favor manipulation using image-based grasp representation. For more general 3D grasping, the full $S E(3)$ grasp pose should be output. The less general approaches employ object shape recognition strategies, where the recognized instances provides strong grasp priors from the object geometry or known grasp strategies relative to the object's pose. Object recognition methods use the known object category and its predicted pose to inform grasping (Tremblay et al. 2018), or use object belief to score grasp quality (Mahler et al. 2016).

Object-agnostic solutions lie in two categories. The first category mainly employs geometric information to approximate the object or robot gripper for 3-D grasp detection. Based on prior object knowledge, modelbased methods approximate objects with box-based shapes (Huebner and Kragic 2008) or primitive surface shapes (Lin et al. 2020) for candidate grasp generation. Once the parametric shape is recognized, extracted, and estimated, the grasps naturally follow. Early model-free methods (Bohg and Kragic 2010) utilized edge features of objects to compute shape context and extract grasp points. Localized antipodal geometry provides an effective means to identify good candidate grasps (ten Pas and Platt 2018; Zapata-Impata et al. 2017). Instead of estimating object geometry, other works focused on the hand contact model for multi-fingered grippers and its role in supporting grasp recognition (Sorour et al. 2019; Kopicki et al. 2016). Sorour et al. (2019) approximated the gripper model by a set of ellipsoids and generated valid grasps via the computed object pose. Kopicki et al. (2016) modeled the contact of hand configurations and surface features using a kernel-density representation, which was used to score grasp candidates.

The second category involves methods that process $3 \mathrm{D}$ point cloud information in either a discriminative or a generative manner. Discriminative methods (ten Pas et al. 2017; Liang et al. 2019) focus on evaluating or scoring grasp hypotheses sampled by a given grasp sampler. Rather than just predict grasp scores, Lou et al. (2020) proposed to further learn grasp pose reachability. Generative methods Qin et al. (2020); $\mathrm{Ni}$ et al. (2020); Wu et al. (2020) performed direct regression of the hypothesized 6-DoF grasp configuration. By introducing post grasp refinement, Mousavian et al. (2019); Zhao et al. (2020) forms a closedloop grasp detection to improve detection accuracy. For addressing 6-DoF grasping detection in complex cluttered scenarios, Murali et al. (2020) proposed to also predict the collision score for grasp candidates. Following the idea of learning the offset, Jeng et al. (2020) proposed a coarseto-fine representation for 6-DoF grasps. To reduce 6-Dof grasp to low-dimensional representation, Sundermeyer et al. (2021) proposed to project 6-DoF grasps to 4-DoF grasp representation conditioned on a known contact point. The grasp representation is composed of 3-DoF grasp rotation and grasp width. It exploits grasp contact geometry to define specific axes capable of recovering the entire grasp geometry from 4 parameters for a given contact point. The approach here employs a similar concept but operates in image-space instead of using a reconstructed point cloud in the vicinity of the candidate grasp. Prior information applied to the 2D image-based grasp geometry reconstructs the 3D grasp geometry.

\section{Problem Statement}

This paper explores the impact of the grasp representation on performance for image-based 2D grasp recognition deep networks, and its impact on the design of the deep network. The grasp recognition problem of interest involves using a color and depth image pair (i.e., RGB-D image) as input to provide as output potential grasp candidates of the form $g=(x, y, \theta, w, h)^{T}$. The deep network output used to obtain $g$ will be that of grasp keypoint pairs. These pairs define the left-middle and right-middle points of a keypoint grasp

$$
g_{\mathrm{kp}}=\left[r_{\mathrm{lm}}^{T}, r_{\mathrm{rm}}^{T}\right]^{T}=\left[x_{\mathrm{lm}}, y_{\mathrm{lm}}, x_{\mathrm{rm}}, y_{\mathrm{rm}}\right]^{T}
$$

in image coordinates, where the subscript $\cdot \mathrm{lm}$ stands for leftmiddle and $\cdot_{\mathrm{rm}}$ for right-middle. For grippers with constant finger widths, the keypoint grasp representation is a simpler, alternative method for representing grasp candidates. It removes from consideration the fixed parameter, $h$, of the bounding box as it is not commonly adjusted in typical scenarios.

After identifying a grasp keypoint pair, as shown in Fig. 3 with $\mathrm{A}$ and $\mathrm{B}$ denoting the two keypoints, conversion of the pair from keypoint grasp coordinates to more standard grasp coordinates

$$
g=(x, y, \theta, w)^{T}
$$




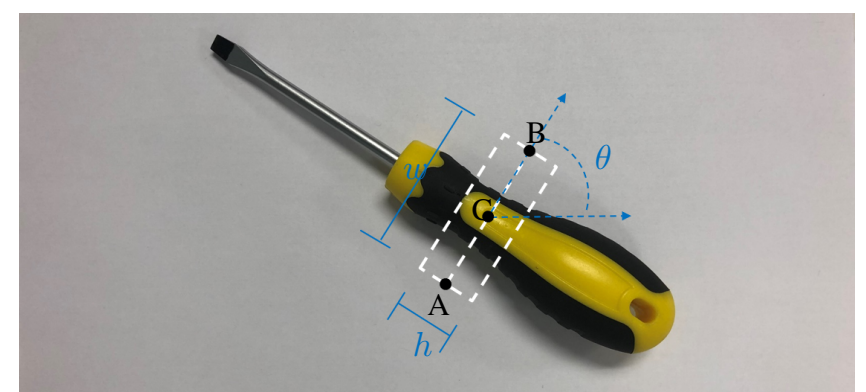

Figure 3. Points $A$ and $B$ are two grasp keypoints. Point $C$ is the center keypoint. The angle $\theta$ is the continuous rotation angle between the line segment $\overline{\mathrm{AB}}$ and the $\mathrm{x}$-axis. The width $w$ of the bounding box is recovered from the length of the segment $\overline{\mathrm{AB}}$. The height $h$ of the bounding box isn't implemented in the proposed representation.

is straightforward. In particular, $r=(x, y)^{T}=\frac{1}{2}\left(r_{\mathrm{lm}}+\right.$ $\left.r_{\mathrm{rm}}\right), \theta=\angle\left(r_{\mathrm{rm}}-r_{\mathrm{lm}}\right)$, and $w=\left\|r_{\mathrm{lm}}-r_{\mathrm{rm}}\right\|_{2}$. Our aim is to show that encoding the image-based, left-middle and rightmiddle representation within a deep network improves grasp prediction performance and robustness with fast processing rates, when it is used to solve grasping as a perception problem and to execute the grasps on robotic manipulators.

\section{Approach}

This section describes the grasp keypoint network, GKNet, as motivated by recent one-stage keypoint detection methods such as CornerNet (Law and Deng 2018) and CenterNet (Duan et al. 2019). The overall neural network architecture of GKNet is depicted in Fig. 2. The backbone deep network is an hourglass type, which has an encoder-decoder structure with iterative and hierarchical skip connections (not depicted in the figure). There are two branches in the network, one to generate grasp hypotheses (top branch) and one to validate them (bottom branch). The grasp hypothesis branch produces two grasp keypoint heatmaps: heatmaps for the left-middle and right-middle grasping points. Each grasp keypoint heatmap has additional data output along with it (e.g., embedding and offset maps) thatserves to refine and constrain the final grasp keypoint options. The validation branch generates one center keypoint heatmap trained to output the center keypoint (i.e., the midpoint between the left-middle and right-middle keypoints).

When implemented as a grasp recognition procedure, the processing flow first uses a neural network trained to predict sets of left-middle, right-middle, and center keypoints over the input image domain (Section 4.1). Additional data output for these keypoints includes embedding values, which support the second step of grouping the left-middle and right-middle keypoints based on embedding value similarity (Section 4.2). The identified keypoint groups are estimated to be valid based on the center keypoint heatmap and a series of conditions for their center keypoints. An orientation filter further refines the final grasp candidate set (Section 4.3). Section 4.4 covers additional details of this process.

\subsection{Keypoint Detection}

4.1.1 The grasp hypotheses branch. To support the generation of grasp hypotheses, the grasp keypoint heatmaps

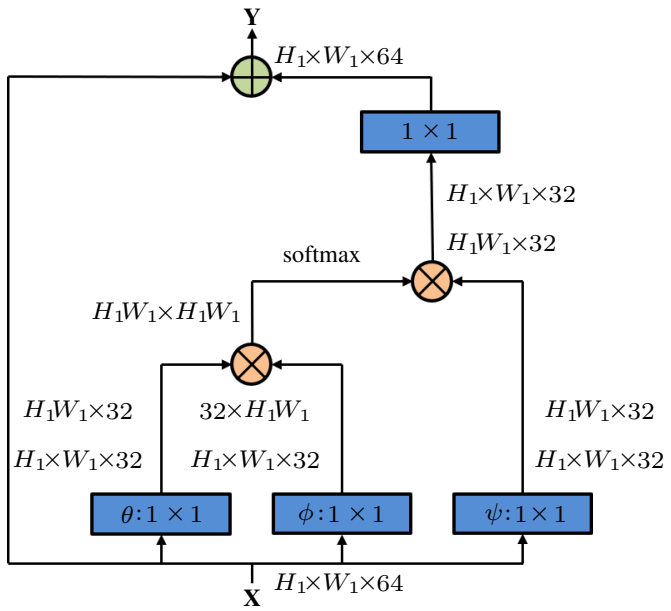

Figure 4. The non-local module architecture. The feature maps are represent by their shapes, e.g., $H_{1} \times W_{1} \times 1024$ where $H_{1}$, $W_{1}$ and 1024 are the height, width and channels. " $\otimes$ " denotes the matrix multiplication, and " $\bigoplus$ " denotes the element-wise addition. The blue boxes represent $1 \times 1$ convolutions.

are defined over image coordinates and a quantized set of grasp orientation classes. Given the symmetry of grasping with parallel plate type grippers, the relevant domain for the grasp angles lies within a 180 degree range: $[-90,90]$ with orientations outside of the domain mapped into it through the modulo operation. Denote by $\mathcal{C}$ the set of orientation classes. Each class element $c \in \mathcal{C}$ represents a quantization interval. The grasp keypoint branch generates two heatmaps $\hat{Y}_{L}^{\mathrm{gk}}, \hat{Y}_{R}^{\mathrm{gk}}: \mathcal{C} \times \mathbb{R}^{2} \rightarrow \mathbb{R}$ stored as matrices $\hat{Y}_{L}^{\mathrm{gk}}, \hat{Y}_{R}^{\mathrm{gk}} \in \mathbb{R}^{|\mathcal{C}| \times H \times W}$ for the left-middle and right-middle keypoints, where $H$ and $W$ are the height and width dimensions of the heatmaps. Each coordinate location indexing into a grasp keypoint heatmap represents the probability distribution over the orientation classes $c \in \mathcal{C}$.

Because the left-middle and right-middle keypoints have a geometric relationship relative to each other and to the graspable region, these heatmaps should be correlated or coupled to implicitly capture important relationships. Per Fig. 2, a non-local module is inserted in the grasp keypoint branch, between the backbone network output and the grasp keypoint heatmap generator input. Non-local modules provide simple and efficient mechanisms to capture longrange spatial dependencies for deep CNNs, dependencies that may not be captured through the standard convolution and pooling operations due to their locality properties (Wang et al. 2018). The module contributes to learning larger scale spatial dependencies that improve the performance of downstream processing. As shown in Figure 4, the input and output dimensions are the same. The intermediate processing within the module generates a response output at a given location as a weighted sum of the features at all locations of the input feature map. The training process couples the outputs at given locations to feature vectors that lie outside of the spatial domain where convolutional and pooling structures can couple image data. From a grasping perspective, the left-middle and right-middle keypoints are spatially separated but geometrically coupled. The intent is for the non-local module to learn feature map dependencies 
that contribute to joint keypoint detection and keypoint grouping.

Besides the keypoint heatmaps, this branch also generates embedding and offset mappings. The offset mappings are the most relevant to keypoint detection, while the embedding is used later for grasp prediction (see Section 4.2). The keypoint heatmaps are output at a lower resolution than the input images, which leads to quantized outputs when the keypoint coordinates in the output domain are mapped to keypoint coordinates in the input image domain. The offset mapping corrects the quantization error due to resolution mismatch between the input image dimensions and the heatmap dimensions. The correction is done by predicting a small coordinate correction to the predicted pixel locations, thereby providing grasp keypoint coordinates with pixellevel resolution in the input image coordinates, as obtained from heatmap maxima in lower resolution coordinates. Denote an offset mapping by $\hat{O}: \mathbb{R}^{2} \rightarrow \mathbb{R}^{2}$, as encoded by the matrix $\hat{O} \in \mathbb{R}^{2 \times H \times W}$. There will be one for each grasp keypoint heatmap, $\hat{O}_{L}$ and $\hat{O}_{R}$, for left and right.

4.1.2 The validation branch. The center keypoint heatmap in the validation branch reflects the probability distribution of grasp midpoints. The grasp midpoint is agnostic to the orientation of the grasp, therefore there are only two classes grasp and no-grasp. Given the opposing probability relationship between these two classes, only a single heatmap is required to capture the distribution (here, it is of the grasp class). The center keypoint heatmap $\hat{Y}^{\text {cen }}: \mathbb{R}^{2} \rightarrow \mathbb{R}$ is stored as the matrix $\hat{Y}^{\text {cen }} \in \mathbb{R}^{H \times W}$.

4.1.3 Network loss functions. Using the same definition for the variant of focal loss used in CornerNet (Law and Deng 2018), define the objective loss functions for the grasp keypoint heatmap and center keypoint heatmap to be

$$
\begin{aligned}
& \mathcal{L}_{\mathrm{det}}^{\mathrm{gk}}(\hat{y}, y)=\frac{-1}{N} \sum_{c=1}^{C} \sum_{i=1}^{H} \sum_{j=1}^{W} L_{\mathrm{det}}\left(\hat{y}_{c i j}, y_{c i j} ; \alpha, \beta\right) \text { and } \\
& \mathcal{L}_{\mathrm{det}}^{\mathrm{cen}}(\hat{y}, y)=\frac{-1}{N} \sum_{i=1}^{H} \sum_{j=1}^{W} L_{\mathrm{det}}\left(\hat{y}_{i j}, y_{i j} ; \alpha, \beta\right),
\end{aligned}
$$

where the heatmap detection loss function is

$$
L_{\operatorname{det}}(\hat{y}, y ; \alpha, \beta)= \begin{cases}(1-\hat{y})^{\alpha} \log (\hat{y}) & \text { if } y=1 \\ (1-y)^{\beta} \hat{y}^{\alpha} \log (1-\hat{y}) & \text { otherwise }\end{cases}
$$

for given hyperparameters $\alpha$ and $\beta$ that affect the penalization of negative samples (we set $\alpha$ and $\beta$ to be 2 and 4 for all experiments). For the grasp keypoint heatmap, the map $\hat{Y}^{\mathrm{gk}}: \mathcal{C} \times \mathbb{R}^{2} \rightarrow \mathbb{R}$, indexed via $\hat{y}_{c i j}^{\mathrm{gk}}$ for the orientation class $c \in \mathcal{C}$ and the location $(i, j) \in \mathbb{R}^{2}$, describes the predicted heatmap score at $(i, j)$ for class $c$, while $y^{\mathrm{gk}}$ : $\mathcal{C} \times \mathbb{R}^{2} \rightarrow \mathbb{R}$ is the corresponding ground truth. Since the center keypoint heatmap has only one class, it is $\hat{Y}^{\text {cen }}$ : $\mathbb{R}^{2} \rightarrow \mathbb{R}$ with $(i, j) \in \mathbb{R}^{2}$ indices specifying the evaluation coordinates. The same holds for the ground truth center heatmap $\hat{y}^{\text {cen }}: \mathbb{R}^{2} \rightarrow \mathbb{R}$. The number $N$ in (3) and (4) is the quantity of grasp bounding boxes in the input image.

Assume that the reduction factor between the input image dimensions and the heatmap dimensions is given by $R$. Let $k$ index into the ground truth keypoint for a given input image.
The pixel coordinates $\left(i_{k}, j_{k}\right)$ of the keypoint determine the ground truth offset vector $o_{i_{k} j_{k}} \in \mathbb{R}^{2}$ for the quantized heatmap coordinates,

$$
o_{i_{k} j_{k}}=\left(\frac{i_{k}}{R}-\left\lfloor\frac{i_{k}}{R}\right\rfloor, \frac{j_{k}}{R}-\left\lfloor\frac{j_{k}}{R}\right\rfloor\right),
$$

where $\lfloor\cdot\rfloor$ represents the floor operation. The objective function for the offset map is the smooth $L_{1}$ loss (Ren et al. 2015), written as $L_{1}(\cdot)$, and leading to the offset map loss functional

$$
\mathcal{L}_{\mathrm{off}}^{\mathrm{gk}}=\frac{1}{N} \sum_{k=1}^{N} L_{1}\left(\hat{o}_{i_{k} j_{k}}, o_{i_{k} j_{k}}\right),
$$

where $\hat{o}_{i_{k} j_{k}}$ is the offset map output value at $\left(i_{k}, j_{k}\right)$ for a given input image and ground truth data containing $N$ keypoints.

\subsection{Keypoint Grouping}

The detection step in the grasp hypothesis branch generates candidate left-middle and right-middle keypoints. To generate grasp candidates, these keypoints must be paired or grouped. Grouping will use the embedding to confirm keypoint pairings. Conceptually, the embedding (Newell et al. 2017) attempts to recover the association between different grasp keypoints in a learnt embedding vector space. When combined with the orientation class predictions and the validation branch, the keypoint grouping step produces ranked grasp candidates.

4.2.1 The embedding. The associative embedding implementation used here was first implemented for detecting human joints through an embedding vector for each joint (Newell et al. 2017). The distance between embeddings relates to the likelihood that different joints are grouped together, and serves as a similarity score. Here, the same process is repurposed to generate embeddings for the leftmiddle and right-middle keypoints with the aim of outputting similar values for paired keypoints and dissimilar values for unpaired keypoints. As a function, the embedding maps the network feature space to a scalar embedding value, $\hat{E}: \mathcal{F} \rightarrow$ $\mathbb{R}$, where $\mathcal{F}$ is the network feature space co-domain of the non-local module. The processing generates multiple leftmiddle and right-middle grasp keypoints associated with the 1D embedding values, each of which has a left-middle and right-middle embedding, denoted $\hat{E}_{L}$ and $\hat{E}_{R}$.

4.2.2 The embedding loss. Learning the embedding involves promoting keypoints belonging to the same grasp to output similar scalar values, while forcing ones belonging to different grasps to output dissimilar values. Since the embedding attempts to connect the spatially separated left-middle and right-middle grasps, the non-local module described earlier can contribute to this coupling by inducing dependencies across all pixels in the feature map. Using competing pull and push losses achieves similarity/dissimilarity learning, such that backpropogating errors through the non-local module induces the necessary dependencies. The pull loss drives the network to predict similar embeddings for keypoints belonging to the same grasp bounding boxes. The push loss drives the network 

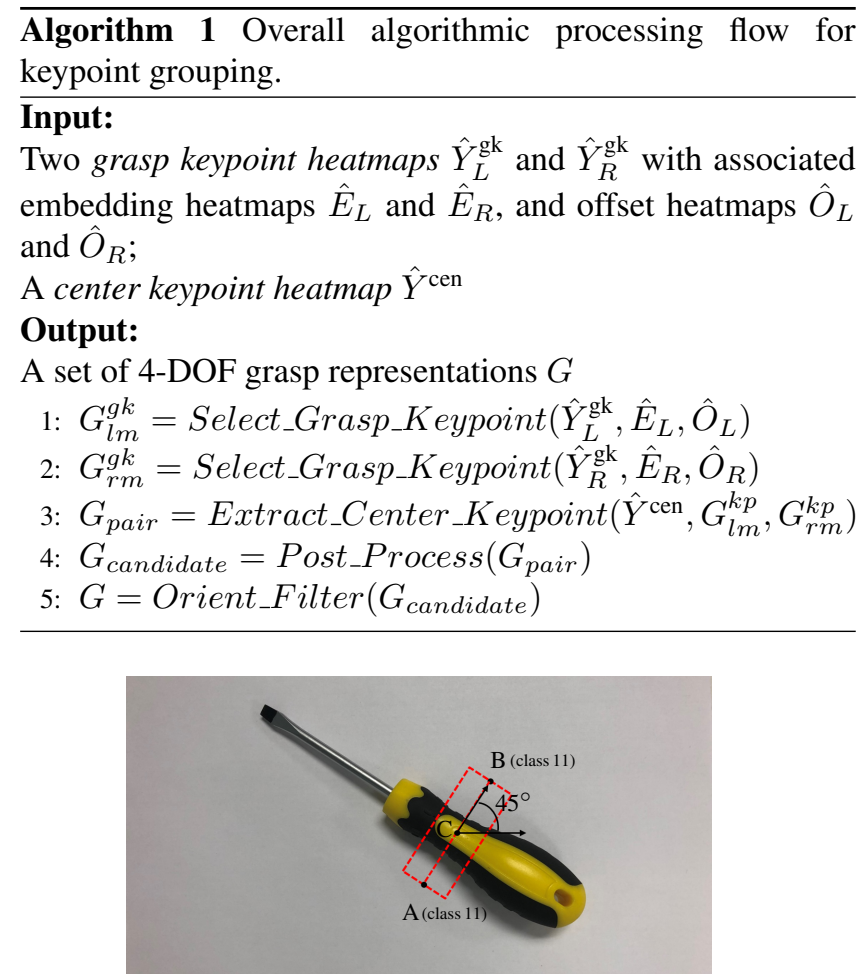

Figure 5. Orientation filtering intends to filter out grasp pairs whose difference between continuous and discrete orientation is over a certain threshold.

to separate keypoints belonging to different grasp bounding boxes, Each grasp $k$ contained in an image from the ground truth dataset has associated to it two hypothesized embedding values $\hat{e}_{L}^{k}$ and $\hat{e}_{R}^{k}$ based on the network's feature vector output $f_{L}^{k}, f_{R}^{k} \in \mathcal{F}$ at that keypoint's location. The pull and push losses are

$$
\begin{aligned}
\mathcal{L}_{\text {pull }}^{\mathrm{gk}} & =\frac{1}{N} \sum_{k=1}^{N}\left[\left(\hat{e}_{l m_{k}}-\hat{e}_{k}\right)^{2}+\left(\hat{e}_{r m_{k}}-\hat{e}_{k}\right)^{2}\right] \\
\mathcal{L}_{\mathrm{push}}^{\mathrm{gk}} & =\frac{1}{N(N-1)} \sum_{k=1}^{N} \sum_{j=1, j \neq k}^{N} \max \left(0,1-\left|\hat{e}_{k}-\hat{e}_{j}\right|\right)
\end{aligned}
$$

where $\hat{e}_{k}$ is the mean value of $\hat{e}_{l m_{k}}$ and $\hat{e}_{r m_{k}}$ and $\hat{e}_{j}$ is the mean value of other keypoint pairs.

4.2.3 Grasps as paired keypoints. When multiple keypoint prediction classes have geometric constraints between them, keypoint estimation benefits from defining an anchoring keypoint, usually defined to be the center or some other center-like location (Duan et al. 2019). The center keypoint heatmap plays that role here. Each pixel on the center keypoint heatmap presents the probability of being a center point for some left-right keypoint pair. Initially grasp keypoint pairs are determined purely by the grasp orientation class and embedding values. Pairs with large differences between the embedding values of the two keypoints will be removed (i.e., less than $\left.\rho_{\text {embed }}\right)$. The center points from the candidate leftmiddle and right-middle keypoint pairs provide a follow-up culling step by obtaining the confidence scores of the center coordinate from the center keypoint heatmap. Pairs leading to a low confidence are removed (i.e., less than $\rho_{\text {cen }}$ ).

\subsection{Orientation Filter}

By quantizing the continuous orientation into discontinuous classes, the multi-class classification of grasp orientation might suffer from discontinuities. Furthermore, performing dense pixel-wise orientation classification might lead to errors through missing global visual information. To address potential issues from the discrete orientations and regional information, a continuous grasp orientation hypothesis is directly computed via grouped keypoints and provides complementary information for comparison. The keypoint grouping process outputs a set of grasp candidates, each represented by the tuple $\gamma=\left(p_{L}, p_{R}, \hat{c}, s\right)$ composed of the left and right grasp keypoints, the shared orientation class, and the center keypoint confidence score, respectively. At this step, there are two distinct forms of orientation information contained in each grasp candidate tuple. One is the discrete orientation $\theta_{1}$ from class $c \in \mathcal{C}$ and the second is the continuous orientation $\theta_{2}$ based on the left and right keypoints,

$$
\begin{array}{rlrl}
\theta_{1} & =\frac{\pi}{|\mathcal{C}|} \hat{c}-\frac{\pi}{2} & \in\left[-\frac{\pi}{2}, \frac{\pi}{2}\right], \\
\theta_{2}=\angle\left(p_{R}-p_{L}\right) & \in\left[-\frac{\pi}{2}, \frac{\pi}{2}\right] .
\end{array}
$$

The orientation filtering step eliminates grasp candidates whose absoluate difference between the discrete and continuous orientations is larger than a threshold, $\tau_{\text {orient }}$. For the candidate grasp shown in Fig. 5, the classification of two grasp keypoints is 11 whereas its continuous orientation is $45^{\circ}$. Presuming that the class label 11 corresponded to an orientation of $30^{\circ}$, then the detection would be discarded due to the large difference between the two orientations. If instead 11 corresponded to $45^{\circ}$, then the grasp would be kept.

\subsection{The GKNet Procedure}

This section describes the Grasp Keypoint Network procedure for collecting keypoint candidates from visual input and then grouping them into grasp candidates, with the steps performed after the network output summarized in Algorithm 1. The grouping process takes as input the grasp keypoint, embedding, and offset heatmaps from the grasp hypotheses branch, and the center keypoint heatmap from the validation branch, then provides a set of grasp representations (2). Step 1 selects the top- $k$ keypoints over the predicted left grasp keypoint heatmap based on the confidence score and extracts the associated embedding values and offsets at the locations of selected keypoints ( $k=$ 100 in all experiments). This step leads to a set of keypoint descriptions given by $G_{\mathrm{lm}}^{\mathrm{gk}}=\left(x_{\mathrm{lm}}, y_{\mathrm{lm}}, o_{x}, o_{y}, c, s, v_{\text {embed }}\right)$ where $x_{l m}$ and $y_{l m}$ are the keypoint coordinates, $o_{x}$ and $o_{y}$ are the coordinate offsets, $c$ is the quantized orientation class, $s$ is the confidence score and $v_{\text {embed }}$ is the embedding value. Step 2 applies the same procedure for the right grasp keypoint heatmap. In Step 3, the selected left and right keypoints combine to create $k^{2}$ pairs. Each pair has a confidence score obtained from the center keypoint heatmap based on the computed location of the center point between the left and right coordinates. Step 4 filters the $k^{2}$ grasp pairs into coarse grasp candidates based on three conditions (1) the orientation classes of the left and right keypoints should agree, (2) the difference between embedding values of the 
left and right keypoints should be less than a given threshold $\rho_{\text {embed }}$, and (3) confidence scores of center points should be above a threshold $\rho_{\text {cen }}$. In Step 5, the remaining grasp candidates go through the orientation filter algorithm with passing candidates forming the set of hypothesized good grasps. The number of output final grasps is set to be 100 in all experiments.

\section{Vision Benchmarks and Evaluation}

The Grasp Keypoint Network approach to grasp recognition is a visual processing approach intended to integrate with a robotic manipulation pipeline. Consequently, evaluation of the approach will involve both benchmarking as a visual input/output method, and as a component of a manipulation system with an actual manipulator. Visual processing will consider the accuracy of the output and the timing of the method relative to other published one-stage, two-stage, grasp quality and other deep learning approaches (Jiang et al. 2011; Asif et al. 2017; Lenz et al. 2015; Mahler et al. 2017; Morrison et al. 2019; Wang et al. 2016; Redmon and Angelova 2015; Kumra and Kanan 2017; Guo et al. 2017; Zhou et al. 2018; Chu et al. 2018; Asif et al. 2019).

Prior to discussing the outcomes of the various experiments, this section describes the GKNet training process, the experimental evaluation criteria, and any additional common methodological elements associated to the vision-only experiments. Experimental setup and evaluation metric for physical experiments will be discussed in Section 7.

\subsection{Datasets and Preprocessing}

There are two benchmark datasets commonly used for training and testing grasp detection networks: the Cornell and Jacquard datasets (Lab 2013; Depierre et al. 2018). The Cornell dataset contains 885 RGB-D images of 244 different objects, with multiple images taken of each object from different poses. The image dataset was collected and manually annotated with 5,110 positive and 2,909 negative grasps. Though the Cornell dataset is used as a standard dataset, annotation is relatively sparse and its size is small. Based on a subset of ShapeNet (Chang et al. 2015), the simulation-based Jacquard dataset has more than 50,000 images of 11,000 objects, with 1 million positive grasps.

5.1.1 Cornell Dataset. Following other RGB-D implementations already trained on pre-existing backbones for a different task (e.g., object detection), we pass to the network the RG-D channels only by using the depth channel as the blue channel input (Redmon and Angelova 2015). The depth information is normalized to lie in the same range, [0,255], as the RGB data. All input channels are re-scaled to lie in the range $[0,1]$, followed by a whitening process (per channel, per pixel, mean value subtraction with division by the standard deviation). The obtained mean values for the RG-D channels are $(0.85,0.81,0.25)$, with standard deviations of $(0.10,0.11,0.09)$. The image resolution and annotation coordinates are remapped to the matrix dimensions $256 \times 256$, followed by application of the data augmentation strategy presented in (Chu et al. 2018) to increase the amount of annotated input/output data and avoid model overfitting.
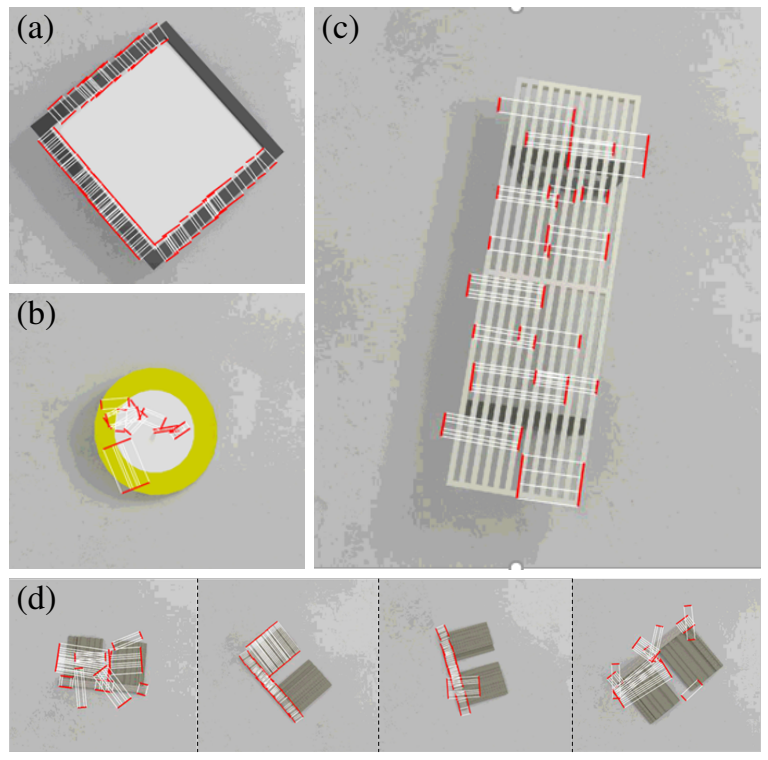

Figure 6. (a)-(d): Four issues with the Jacquard dataset.

5.1.2 Jacquard Dataset. The Jacquard dataset was produced through automatically generated annotations in order to have a much larger training set relative to the Cornell dataset without the manual annotation demands. Done in simulation, the grasps were obtained via a sample and confirm process. Compared with the Cornell dataset, images in the Jacquard dataset are annotated with much denser grasping bounding boxes, however they are not all physically meaningful. While the Cornell dataset has many missing (false negative) annotations, the Jacquard dataset has several false positive annotations as well as missing annotations. After reviewing the dataset, we identified the following four problems with the annotations: (1) They can be dense on one side but sparse or even missing on the other side for some objects with symmetric shapes, see Fig. 6 (a); (2) Some do not seem plausible and may be a function of incorrect physics within the simulator, see Fig. 6(b); (3) The sampling process can lead to sparse annotations for certain objects with multiple, continuous grasp regions, see Fig. 6(c), (4) Orientation dependent grasp annotations lead to highly variable grasp annotation outcomes for the same object, Fig. 6(d). These issues negatively influence the training process and the test evaluation processes.

To correct for these issues, we defined a filtering strategy for selecting a subset from the Jacquard dataset. The filter strategy follows three rules based on the ratio of the union of all grasp regions to the masked region of the object: (a) Keep annotation data whose ratio is larger than 0.8 ; (b) Remove annotation data whose ratio is less than 0.2 ; and (c) Manually check images whose ratio is between 0.2 and 0.8 and remove those images whose annotations satisfy either one of the above four cases. We call the resulting selected subset the Abridged Jacquard Dataset (AJD), which is publicly available (Xu et al. 2020a). The AJD consists of 46,857 images of 10,799 objects, with $4,420,953$ positive grasp bounding boxes in total.

Due to downsampling of output keypoint heatmaps relative to the input image, multiple grasp keypoints from different grasp instances can map to the same pixel in the ground truth heatmap. For example, if the left-middle point 
of grasp instance $A$ is within the radius of downsampling ratio of the left-middle point of grasp instance $B$ on the input image, they will be located at the same pixel on the groundtruth heatmap. To deal with the resulting ambiguity, we refine the dense annotations by always selecting the first grasp bounding box processed to make sure that there will not be any points from different grasp bounding boxes mapping to the same pixel on the downsampled map. The above strategy isn't applied to the Cornell dataset, since its grasp annotations are sparse.

Image normalization follows the same procedure applied to the Cornell dataset. The mean values of the RG-D channels are $(0.71,0.71,0.20)$, and the standard deviations are $(0.06,0.07,0.09)$. The Jacquard images and annotations were remapped to the matrix dimensions $512 \times 512$, with no data augmentation applied.

\subsection{Deep Network Architecture and Training}

We use Deep Layer Aggregation (DLA) as our backbone network to extract features from input images ( $\mathrm{Yu}$ et al. 2018). DLA augments standard neural network architectures with an iterative and hierarchical aggregation strategy to fuse semantic and spatial information across layers. The deep layer aggregation structures helps network capture deeper and wider information for recognition and localization. Meanwhile the hierarchy aggregation architecture requires fewer parameters, which reduces size of the entire network and inference time. We choose DLA as our final backbone network since it achieves the best performance. Rich semantic and spatial information improves performance of keypoint detection and fewer network parameters enables GKNet to run in real-time. However there is no restriction on the backbone network for GKNet; it can be switched to other state-of-the-art keypoint detection neural networks.

Our work is implemented in PyTorch 1.1.0 (Paszke et al. 2017) and the backbone network DLA is pretrained on ImageNet (Deng et al. 2009). We use random affine transformation as data augmentation and use Adam (Kingma and $\mathrm{Ba} 2015$ ) to optimize training loss:

$$
\mathcal{L}=\mathcal{L}_{\mathrm{det}}^{\mathrm{gk}}+\mathcal{L}_{\mathrm{det}}^{\mathrm{cen}}+\alpha \mathcal{L}_{\mathrm{pull}}^{\mathrm{gk}}+\beta \mathcal{L}_{\mathrm{push}}^{\mathrm{gk}}+\gamma \mathcal{L}_{\mathrm{off}}^{\mathrm{gk}},
$$

where $\alpha, \beta$ and $\gamma$ are the weights of the pull, push and offset loss for grasp keypoint heatmap. For training, we use a batch size of 8 and train our ntwork end-to-end on a single Titan XP. Training a network model with the Cornell dataset runs for 20 epochs with an initial learning rate of $1.25 \times 10^{-4}$ that gets divided by 10 at the 10th and the 15 th epoch. Training a network model with the AJD runs for 30 epochs with the same initial learning rate and with divisions at the 20th and the 25 th epoch.

\subsection{Parameters}

We report some essential hyper-parameters used in training and testing process here; the rest are available in the released code (Xu et al. 2020b). With a downsampling ratio of $R=4$, GKNet outputs heatmaps with size of $57 \times 57$ and $128 \times 128$, for the Cornell and Jacquard dataset respectively. For all training processes, we choose weighting coefficients in (12) to be $\alpha=1, \beta=1$ and $\gamma=1$. Due to the different image source and grasp annotation distributions, we set different testing stage filtering coefficients $\rho_{\text {embed }}, \rho_{\text {cen }}$ and $\tau_{\text {orient }}$ for the datasets.

Cornell. We set $\rho_{\text {embed }}=1.0, \rho_{\text {cen }}=0.05$ and $\tau_{\text {orient }}=$ 0.24 . The number of orientation classes is $|\mathcal{C}|=18$.

AJD. We set $\rho_{\text {embed }}=0.65, \rho_{\text {cen }}=0.15$, and $\tau_{\text {orient }}=$ 0.1745 . The number of orientation classes is $|\mathcal{C}|=36$.

\subsection{Evaluation Metrics for Vision Benchmarks}

The standard grasp evaluation metric considers the full grasp rectangle (Lenz et al. 2015). By design, the GKNet grasp representation lacks the variable $h$ describing the bounding box. To apply the same evaluation metric as other publications, the value $h$ is set to the average of all grasp candidates for the training dataset used. These values work out to be 23.33 and 20 for the Cornell dataset and the AJD, respectively. The predicted grasp candidate is a positive match if:

1. the orientation difference between predicted grasp and corresponding ground truth is within $30^{\circ}$; and

2. the Jaccard index of the predicted grasp $g_{p}$ and the ground truth grasp $g_{t}$ is greater than 0.25 :

$$
J\left(g_{p}, g_{t}\right)=\frac{\left|g_{p} \cap g_{t}\right|}{\left|g_{p} \cup g_{t}\right|}>\frac{1}{4} .
$$

The speed of the grasp recognition process is measured in frames per second (fps).

\section{Vision Benchmark Results}

Assessment of GKNet as a visual processing algorithm tests two aspects: the network design choices and the final design for grasp prediction. Regarding design elements, there is the assertion that the two keypoint representation with left-middle and right-middle keypoints is a preferred representation over alternative versions (\$6.1). Secondly, different backbone options are possible with similar properties (\$6.2). The two studies will lead to the final chosen design for GKNet. Benchmark evaluations will explore the hypothesis that a keypoint-based, one-stage neural network structure can provide the best outcome relative to performance and processing speed. GKNet is compared to other published works using the Cornell dataset (§6.3), and with publicly available methods using the AJD (§6.4). Visualizing published methods on a performance versus processing frame rate graph shows that GKNet is closer to the extremal corner (high performance and high frame rate) than other methods, thereby confirming the assertion of balancing accuracy and speed.

\subsection{Grasp Keypoint Representation Study}

There is some flexibility when choosing the keypoint representation for recovering grasp bounding boxes, four of which are depicted in Fig. 7. The first two preserve the full grasp representation coordinates, while the last two provide less information related to the lengths of the bounding box edges. To confirm that the chosen middle points representation provides the best outcomes, grasp keypoint networks with the other representations were also created and trained using both the Cornell dataset and AJD, 

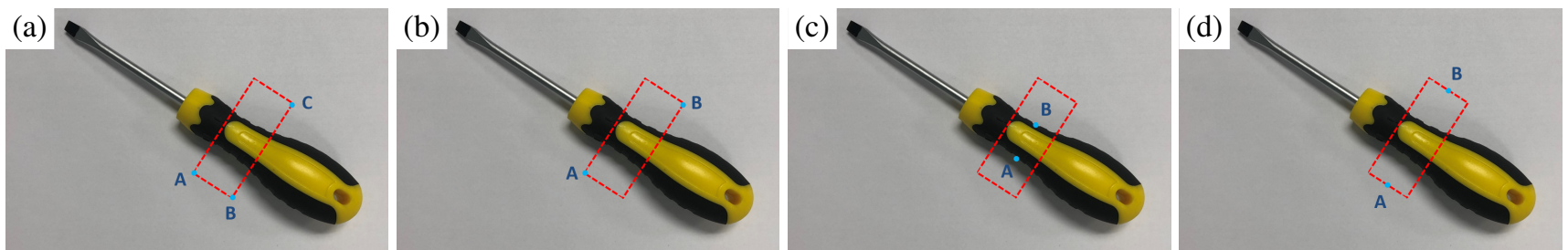

Figure 7. (a)-(d): Grasp bounding box representations with three corner points, two corner points, two edge points, and two middle points. Benchmarking on the AJD indicates that using the two middle points provides the best outcomes.

Table 1. Evaluation of different grasp representations on the Cornell and AJD. TIBIBr: top-left, bottom-left and bottom-right keypoints; TIBr: top-left and bottom-right keypoints; TIBrOri: top-left and bottom-right keypoints with orientation offset; LeRe: left-edge and right-edge keypoints; and LmRm: left-middle and right-middle keypoints.

\begin{tabular}{|c|c|c|c|c|c|}
\hline Rep. & TIBIBr & TIBr & TIBrOri & LeRe & LmRm \\
\hline & \multicolumn{5}{|c|}{ Prediction Accuracy (\%) } \\
\hline Cornell & 94.50 & 92.41 & 85.29 & N/A & 96.90 \\
\hline AJD & 95.89 & 96.38 & 95.34 & 97.02 & 98.39 \\
\hline
\end{tabular}

individually. Since the Cornell dataset does not provide segmentation masks, the two edge points representation, Fig. 7(c), cannot be trained due to missing ground-truth information. Hence, the missing entry for LeRe in Table 1 for outcomes using the Cornell dataset.

There are 4 different grasp representations and one more variation based on the grasp representation in Fig. 7(b), which yields 5 outcomes for each dataset in the Table 1 . When using three corner points, Fig. 7(a), the grouping process will be more complex since additional constraints or factors must be included to properly group the point triplet from the heatmap outputs. They introduce an additional process that can add false positive and negative outcomes. For the top-left and bottom-right corner points, Fig. 7(b), there is no unique bounding box due to the unknown slope of the sides. Here, the estimated slope or orientation of the bounding box edges must be inferred from the discrete orientation classification information in the output grasp keypoint heatmaps. Considering that employing classification to recover orientation inevitably introduces quantization error, we explore a variation to address the quantization error, which incorporates one more branch to predict another heatmap for the orientation offset. The final offset for grouped grasp candidate will be computed by averaging the offset predicted for each grasp keypoint. The third representation with left-edge and right-edge points, Fig. 7(c), was explored as an option based on the premise that object edge points and their neighboring regions contain potentially useful visual information, which could help the network learn visual features of grasp keypoints and support generalization. It contains partial information regarding the bounding box edge lengths (a minimum value for one edge and no information for the other). The fourth representation is the one chosen for GKNet.

All outcomes are recorded in Table 1 . The best performing grasp representation on the Cornell dataset is the LmRm representation. The same holds for the AJD. For the other representations, the Cornell and AJD have opposing trends. For the Cornell dataset, when excluding the LmRm options, performance drops from left to right. For AJD it roughly increases if the TIBrOri option is ignored. The discrepancy may be a result of the Cornell dataset having sparser annotations than the AJD. The drop for TlBrOri relative to $\mathrm{TlBr}$ for both datasets suggests that this orientation ambiguous solution cannot be enhanced by additional orientation information. The performance drop shows the difficulty of learning to directly regress small orientation offsets in these circumstances; paired corner representations and angle quantizations should be avoided.

Focusing on the AJD results, one inference is that keypoint grouping plays an important role in the grasp keypoint detection. Performance lowers with more keypoints, which has the added difficulty of correctly grouping detected keypoints. The performance boost between the TLBr and LeRe representations for AJD shows implicitly encoded rotation information contributes to grasp bounding box estimation and that the quantization error introduced by classification degrades grasp detection.

The LmRm representation has the smallest set of geometric constraints to impose and the most amount of orientation information contained in the network outputs. Both properties contribute to the improved performance. The simplified representation is possible because the edge length variable $h$ was removed from the grasp coordinate vector.

\subsection{Ablation Studies}

Three ablation studies performed are described here. First, several deep neural network backbones are selected to replace the feature extractor of the proposed network. The goal of this test is to evaluate the effectiveness and robustness of our proposed keypoint-based grasp representation across backbone network architectures. Second, for the purpose of evaluating the orientation filter, each trained model will be tested with and without the proposed orientation filter. Third, to evaluate the effectiveness of lower resolution heatmaps with offset mappings, the proposed method will be trained with a variant that predict grasp keypoint heatmaps of the same dimenions as the input image.

Backbone Study. Various backbone options are possible. The performance impact should not vary much since many networks have good learning properties. However, the execution speed and training properties will differ based on the complexity of the network. Five network types were tested, Deep Layer Aggregations (DLA) (Yu et al. 2018) (size 34), Hourglass (Newell et al. 2016) with two sizes (52 and 104), ResNet (He et al. 2016) with two sizes (18 and 50), VGG-16 (Simonyan and Zisserman 2015), and AlexNet. AlexNet, VGG-16 and ResNet are chosen for comparison due to their contributions to the evolution of convolutional 
Table 2. GKNet Backbone: Cornell Dataset

\begin{tabular}{|l|c|c|c|}
\hline Approach & Acc. w o.f. (\%) & Acc. w/o o.f. (\%) & Speed (fps) \\
\hline Alexnet & 95.0 & 94.8 & 83.33 \\
\hline VGG-16 & 96.8 & 96.4 & 55.56 \\
\hline Resnet-18 & 96.0 & 95.7 & 66.67 \\
\hline Resnet-50 & 96.5 & 96.4 & 52.63 \\
\hline Hg-52 & 94.5 & 93.6 & 33.33 \\
\hline Hg-104 & 95.5 & 95.3 & 21.27 \\
\hline DLA & $\mathbf{9 6 . 9}$ & $\mathbf{9 6 . 8}$ & $\mathbf{4 1 . 6 7}$ \\
\hline
\end{tabular}

neural networks. AlexNet uses Rectified Linear Units (ReLu) in place of the tanh function. VGG-16 use $3 \times 3$ filters to replace the large convolution filters in the intial layers and had increased depth. ResNet use a simple skip connections strategy and has fewer layers relative to other contemporary networks. Hourglass-52 and Hourglass-104 (Hg-52 and Hg104) are chosen to explore any potential performance boosts due to a deeper and more connected network, at the cost of inference speed. There are two depth types chosen for ResNet and Hourglass in order to explore the effect of depth for the same network architecture. All models were tested with two variants: with and without the orientation filter component (§4.3).

Table 2 contains the outcomes for the Cornell dataset (image-wise split) and Table 3 contains the outcomes for the Abridged Jacquard dataset. All models are ordered based on their release date. First, they show that the DLA backbone is both accurate and efficient. Runtimes close to or surpassing the typical camera frame-rate $(\sim 20 \mathrm{~Hz})$ are possible. Second, among AlexNet, VGG-16 and ResNet, AlexNet achieves the worst performance. Models of VGG-16 and ResNet achieve similar performance. This result shows that skip connection might not lead to significant improvement in keypoint detection. Third, the improvements from ResNet18 to ResNet-50 and Hourglass-52 to Hourglass-104 show that deeper network architectures help capture rich image features and improve the detection accuracy. However, the speed loss may outweight the performance boost Fourth, Hourglass models do not achieve strong performance compared to other networks with relatively simpler designs. The Hourglass network is composed of a large amount of skip connections from shallow to deep layers, which indicates that skip connecting low-dimensional features might not lead to positive effects. Lastly, the performance difference between the best and worst ones is less than $2.4 \%$ and $1.1 \%$ for the Cornell dataset and the AJD, respectively, relative to the best performing architecture. Networks with simple feature extractors, i.e., AlexNet, still achieve satisifactory results. Limiting to earlier networks, Resnet-50 backbone and those in the rows above, the processing speed mostly increases but with a small decrease in performance; less than $2 \%$ for the Cornell data set and less than $1 \%$ for the AJD. The close performance across all models shows that the proposed grasp keypoint representation is efficient for grasp detection and robust to network architecture.

Orientation Filter. As shown in the Tables 2 and 3, the orientation filter does improve performance by a small amount over all models.

Heatmap Resolution. Implementing GKNet without the offset mapping and with keypoint output at the same
Table 3. GKNet Backbone: Abridged Jacquard Dataset

\begin{tabular}{|l|c|c|c|}
\hline Approach & Acc. w o.f. (\%) & Acc. w/o o.f. (\%) & Speed (fps) \\
\hline Alexnet & 97.37 & 94.53 & 34.48 \\
\hline VGG-16 & 98.36 & 96.13 & 21.28 \\
\hline Resnet-18 & 97.95 & 95.97 & 31.25 \\
\hline Resnet-50 & 98.24 & 95.91 & 25.00 \\
\hline Hg-52 & 97.21 & 93.81 & 15.87 \\
\hline Hg-104 & 97.93 & 96.04 & 9.90 \\
\hline DLA & $\mathbf{9 8 . 3 9}$ & $\mathbf{9 6 . 9 9}$ & $\mathbf{2 3 . 2 6}$ \\
\hline
\end{tabular}

resolution as the input leads to grasp recognition accuracy of $94.0 \%$ and $97.77 \%$ for the Cornell dataset and the AJD, respectively. These values reflect reduced performance relative to the baseline implementation. Upsampling the output too much relative to the initial output is not advisable. A benefit of the lower resolution heatmaps is a faster inference speed due to the reduced computation, plus a smaller memory footprint used during training, even with the offset maps included.

For the remainder of the paper, GKNet will refer to the variant with the DLA-34 backbone, $R=4$, and the orientation filter.

\subsection{Evaluation on the Cornell Dataset}

We first evaluate our work on the Cornell dataset with image-wise and object-wise splits, following the approach of splitting training and testing sets described in Chu et al. (2018). The Cornell dataset is older than AJD and has more published performance outcomes, permitting comparison with a larger set of grasp detection methods. In the comparison, we divide state-of-the-art works into one-stage approaches, two-stage approaches, methods based on grasp quality scores, and other works. Table 4 collects the prediction accuracy for image-wise and object-wise splittings, as well as inference speed. All results are taken from corresponding publications except those of Morrison et al. (2019), which is re-implemented based on the public repository. For Chu et al. (2018), the ResNet-50 results are recorded in Table 4. Most of the published works have a prediction accuracy lower than GKNet, however their inference time is nearly an order of magnitude larger. Focusing on the image-wise split, GKNet accuracy is $0.8 \%$ lower than the best one-stage approach but runs 5 times faster. While also lower by $0.8 \%$ than the best two-stage method, it is 10 times faster. Similar accuracy statistics hold for the object-wise split.

Some of the backbone networks for these methods are older ones, like VGG-16 or ResNet-50. The results in Table 2 indicate that GKNet's performance continues to best those whose accuracy is below $96.0 \%$, irrespective of the backbone chosen (so long as it is not AlexNet). The backbone of Morrison et al. (2019) is similar to AlexNet, but does not perform as well as GKNet w/AlexNet backbone. Thus, the ablation study leading to Table 2 suggests that GKNet's good relative performance is more a function of the representation than the backbone network.

To gain a better sense for how GKNet optimizes for both accuracy and speed, Fig. 8 plots image-wise prediction accuracy versus inference speed for the ten methods from Table 4 for which such information is available. The preferred location for an implementation that jointly 
Table 4. Evaluation on the Cornell Dataset

\begin{tabular}{|l|c|c|c|}
\hline Approach & \multicolumn{2}{|c|}{ Accuracy (\%) } & speed \\
\hline & $\begin{array}{c}\text { image } \\
\text { split }\end{array}$ & $\begin{array}{c}\text { object } \\
\text { split }\end{array}$ & fps \\
\hline other work & & & \\
\hline Jiang et al. (2011) & 60.5 & 58.3 & 0.02 \\
\hline Asif et al. (2017) & 88.2 & 87.5 & - \\
\hline grasp quality & & & \\
\hline Lenz et al. (2015) & 73.9 & 75.6 & 0.07 \\
\hline Mahler et al. (2017) & 93.0 & - & - \\
\hline one-stage & & & \\
\hline Morrison et al. (2019) & 78.4 & 84.3 & 233.73 \\
\hline Wang et al. (2016) & 81.8 & - & 7.10 \\
\hline Redmon and Angelova (2015) & 88.0 & 87.1 & 3.31 \\
\hline Kumra and Kanan (2017) & 89.2 & 88.9 & 16.03 \\
\hline Guo et al. (2017) & 93.2 & 89.1 & - \\
\hline Zhou et al. (2018) & 97.7 & 96.6 & 8.51 \\
\hline GKNet & 96.9 & 95.7 & 41.67 \\
\hline two-stage & & & \\
\hline Chu et al. (2018) & 96.0 & 96.1 & 8.33 \\
\hline Asif et al. (2019) & 97.7 & - & 4 \\
\hline
\end{tabular}

Table 5. Backbone Network Comparison

\begin{tabular}{|l|c|c|}
\hline Approach & Network & Parameters \\
\hline other work & & \\
\hline Jiang et al. (2011) & - & - \\
\hline Asif et al. (2017) & - & - \\
\hline grasp quality & - & - \\
\hline Lenz et al. (2015) & - & $0.18 \mathrm{M}$ \\
\hline Mahler et al. (2017) & & \\
\hline one-stage & - & $0.02 \mathrm{M}$ \\
\hline Morrison et al. (2019) & - & - \\
\hline Wang et al. (2016) & - & $0.67 \mathrm{M}$ \\
\hline Redmon and Angelova (2015) & ResNet-50 & $25.56 \mathrm{M}$ \\
\hline Kumra and Kanan (2017) & ZF-Net & $5.30 \mathrm{M}$ \\
\hline Guo et al. (2017) & ResNet-101 & $44.55 \mathrm{M}$ \\
\hline Zhou et al. (2018) & DLA-34 & $15.74 \mathrm{M}$ \\
\hline GKNet & & \\
\hline two-stage & ResNet-50 & $25.56 \mathrm{M}$ \\
\hline Chu et al. (2018) & DenseNet* & - \\
\hline Asif et al. (2019) & & \\
\hline
\end{tabular}

optimizes accuracy and speed will be near the top-right corner of the figure. GKNet is the approach closest to the top-right corner, which indicates that it achieves a good trade-off between prediction accuracy and inference speed. One factor affecting this conclusion is the different hardware configurations of the methods. To address this factor, GKNet was run on an NVIDIA GeForce GTX 1070 and achieved $37.04 \mathrm{fps}$. Its location in the graph would be close to the plotted GKNet location, which means that GKNet still provides a favorable trade-off between detection accuracy and inference speed on less capable hardware. Being able to maximize both is advantageous. Strong prediction accuracy should translate to high success rate when integrated into a manipulation pipeline. Later experiments will show that the GKNet achieves top ranked success rates in eye-to-hand static grasping experiments relative to other published experiments, with a small performance gap between visual and experimental benchmarking. Low inference time supports robust grasping when the robot executes tasks in dynamic settings (to be shown in Sec. 8).

To get a sense for the backbone network architecture of GKNet relative to existing methods, Table 5 collects

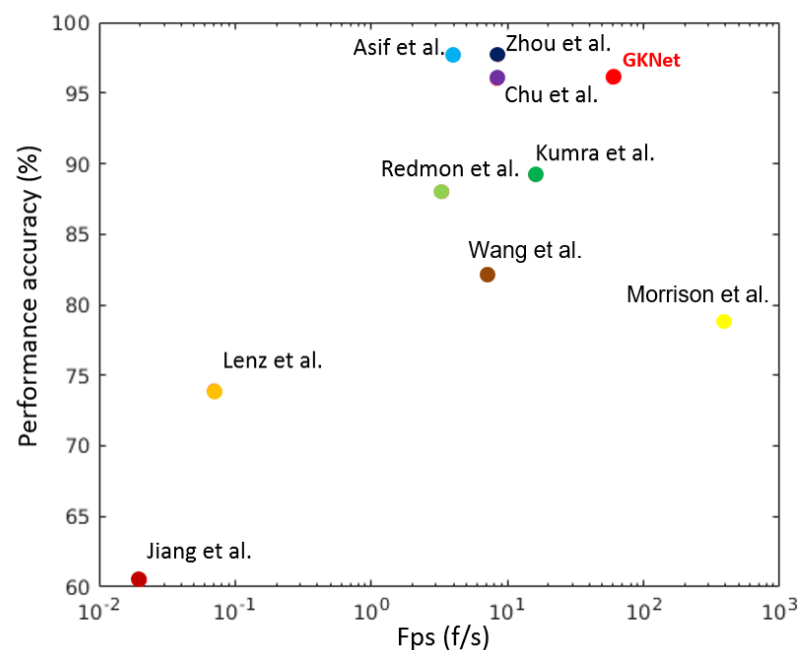

Figure 8. Plot of performance accuracy (\%) versus inference speed (fps) using the Cornell benchmark for published works. Performance accuracy is tested on image-wise split. Inference speed is in log-space. Closer to top-right is better.

Table 6. Evaluation on the Abridged Jacquard Dataset

\begin{tabular}{|l|c|c|}
\hline Approach & Accuracy (\%) & Speed (fps) \\
\hline one-stage & & \\
\hline Morrison et al. (2019) & 86.41 & 250.78 \\
\hline GKNet & $\mathbf{9 8 . 3 9}$ & $\mathbf{2 3 . 2 6}$ \\
\hline two-stage & & \\
\hline Chu et al. (2018) & 95.08 & 7.52 \\
\hline
\end{tabular}

the number of trainable parameters for various backbone networks used in published works. For those methods which do not employ off-the-shelf standard networks for extracting features or do not provide detailed network architectures, corresponding results are left empty. For Redmon and Angelova (2015), Mahler et al. (2017), and Morrison et al. (2019) which do not employ standard off-the-shelf networks, the backbone network parameter counts are computed based on the network architectures described in the publications. For Asif et al. (2019), their base network is a variant of DenseNet but the number of parameters isn't provided. As shown in Table 5, DLA-34, which contains fewer parameters via efficient skip connections structures, contributes to the state-of-the-art trade-off between accuracy and speed. Interestingly, Mahler et al. (2017) achieves close result with a backbone network that is 100 times smaller, which indicates that standard state-of-the-art networks might be too deep and contain redundant parameters.

\subsection{Evaluation on the AJD}

Benchmarking with the AJD dataset will be more limited since the newer Jacquard dataset has less published evaluations. Comparison methods obtained from publicly released code consist of a one-stage detector (Morrison et al. 2019) and a two-stage detector (Chu et al. 2018). For both compared approaches, we employed the same training strategies they released for the Jacquard dataset to train on the AJD. Processing speed estimates were obtained from executing the approaches on the same system hardware. Table 6 contains the evaluation of outcomes for the two baseline methods. GKNet performance remains high and 
outperforms the published methods. The slightly higher performance on the Abridged Jacquard dataset is most likely due to the dense grasp annotations. The inference speed is lower but still close to frame-rate.

\section{Physical Experiments and Evaluation}

This section introduces the experimental setup and evaluation metric for all physical experiments. Manipulation experiments will include several different test and evaluation methods found in the literature or intended to demonstrate functional characteristics. They are: (1) static grasping, (2) dynamic grasping, and (3) grasping at varied camera angles and (4) bin picking. Static grasping is a simple test requiring precise grasp detection for isolated objects, and is commonly performed to establish upper bounds on performance due to the lack of distractors and impediments to grasping. It also tests robustness to object type when many different objects are tested as part of the protocol. Dynamic grasping experiments test the combination of accuracy and speed, but require some additional outer-loop elements for converting the grasp detection system into a grasp tracking one. The experiments primarily test for speed, with the resulting accuracy gap relative to static grasping also being an indication of grasp detection consistency. Grasping under varied camera angles tests the robustness with respect to viewpoint and demonstrates some form of generalization of the algorithm relative to this factor. The bin picking experiment is the most challenging of the four since grasp detection pipelines require additional outer-loop and post-processing elements to identify a preferred grasp. Unlike grasp quality networks, which can be trained under these circumstances, one-stage and two-stage detectors are usually not trained to work in these settings. Some grasp quality networks benefit from simulation for amassing very large ground-truth data, which can be several orders of magnitude larger than for manually annotated datasets (i.e., DexNet grasp data is three orders of magnitude more than the Cornell benchmark dataset). Conducting this experiment will involve introducing a simple grasp prioritization scheme through a model-based grasp scoring system.

\subsection{Robotic Platform Setup}

Our robotic platform setup consists of a Kinect Xbox 360 for image acquisition and a 7 degree-of-freedom custom-made robotic arm for performing grasping, lifting, and placing tasks. The camera setup is an eye-to-hand viewpoint. The distance from the camera center to table is around $1.2 \mathrm{~m}$ for all experiments. The custom-made 7 degree-of-freedom robotic arm, shown in Figure 12, employs DYNAMIXEL servo motors as actuators and is attached with the custommade parallel-jaw gripper, which can be viewed in Figure 11 (a). The gripper width is $17 \mathrm{~mm}$ and the maximal open width is $200 \mathrm{~mm}$. The maximal depth is $80 \mathrm{~mm}$ when the gripper closes.

\subsection{Objects Sets for Manipulation Experiments}

Though there are published robotic benchmark object datasets like the ACRV Picking Benchmark (APB) and the YCB Object Set (Calli et al. 2015b; Leitner et al. 2017), most published works employ their own objects or a subset of objects from these datasets. For comparison purposes, we employ 5 object sets for 4 different kinds of robotic grasping experiments. The test sets include novel objects not included in the training datasets. They are described below and visualized in Fig. 9. They have some overlap with the APB and YCB sets.

Household Sets. The two different household sets are shown in Fig. 9(a) and 9(b), as described in Morrison et al. (2019) and Chu et al. (2018). The Morrison set contains 12 common household objects of different sizes and shapes. The objects were chosen from the standard robotic grasping datasets (Calli et al. 2015b; Leitner et al. 2017). The Chu set contains ten commonly seen objects collected from the Cornell dataset.

Adversarial Set. The adversarial set, shown in Fig. 9(c), consists of eight 3D-printed objects described in Dex-Net 2.0 (Mahler et al. 2017). The objects are characterised by complex and adversarial geometric features such as smooth and curved surfaces, which increase the difficulty of grasping. They are meant to undermine grasp scoring systems. It is less clear what impact these shapes have on other learning-based grasping approaches.

Compound Set. The compound set, shown in Fig. 9 (d), is composed of seven common household objects and 3 objects from the adversarial set.

Bin Picking Object Set. The bin picking object set, shown in Fig. 9(e), is collected for bin picking experiments. The object set has 50 objects consisting of household objects and adversarial objects.

\subsection{Evaluation Metrics for Manipulation Experiments}

Unless otherwise stated, the evaluation criteria for the grasping experiments will consist of the grasping success rate, which is the percentage of successful grasps relative to the total amount of grasp attempts. A grasp is considered to be a success if the manipulator grasps, lifts, and holds the object in the air for three seconds. While some researchers also measure-and advocate for-mean picks per hour (Mahler et al. 2016), we elect to not include this quantitative measure as it is influenced more by the manipulator properties, the manipulation code, and the programmed grasping procedure, than by the visual processing algorithm. In short, within the perception, planning, and action loop, the planning and action steps determine the pick rate more so than the perception step. This manuscript's scope is related to the perception process, not to the planning and action processes.

\subsection{Trained Models for Manipulation Experiments}

For the majority of the manipulation experiments, the Cornell dataset was the training dataset. This permits evaluation of approaches that trained and tested against Cornell, and also performed manipulation experiments. Some methods have a performance gap when moving from visual processing to embodied grasping, which network training data consistency permits comparing with.

The bin picking experiment is the exception, for which GKNet was trained with the AJD. Bin picking scenarios 

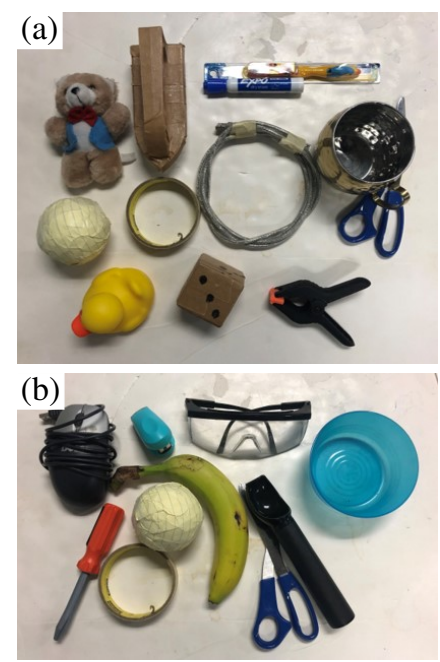
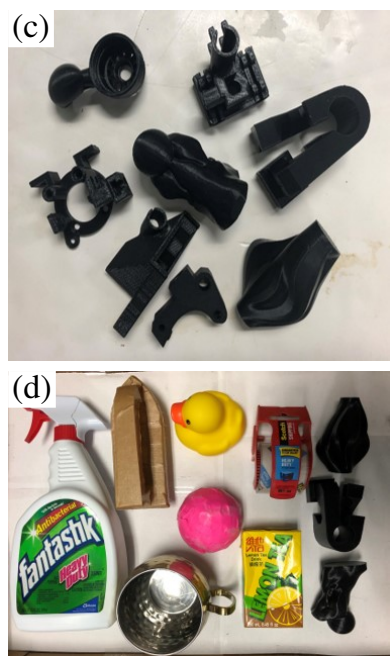

(e)

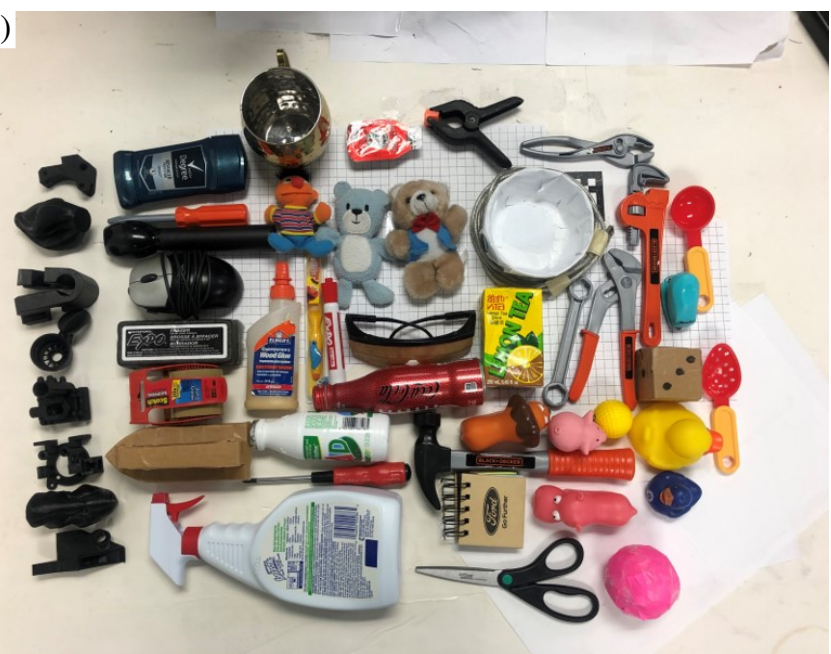

Figure 9. Object sets used for two grasp experiments. (a) The household set (Morrison). (b) The household set (Chu). (c) The adversarial set. (d) The compound set. (e) The bin picking object set.

involve multiple objects, for which neither the AJD nor the Cornell dataset has the equivalent type of training data; both consist of scenes with a single object only. The choice to use AJD is due to differences in the annotation sparsity, or in the ground-truth grasp distributions, between the Cornell dataset and the AJD. The denser annotations in the AJD dataset better aid in locating grasps for object sub-parts, whereas models trained on the Cornell dataset tend to provide grasp candidates with respect to the whole object. For cluttered environments with overlaid or occluded objects, the ability to focus on sub-parts improves the ability to differentiate occlusion geometries and target object regions.

\section{Robot Benchmark Results}

To confirm that the vision-based outcomes translate to equivalent manipulation performance for embodied robotic arms, this section describes the outcomes of the four eyeto-hand manipulation experiments described in Section 7. Specific details regarding the experimental setup and testing approach are included, with comparison to published works where possible.

The aim is to evaluate GKNet with regards to accuracy, speed, and robustness. Here robustness will be with regards to variation in object categories, or with respect to a nuisance factor (object motion, camera viewpoint, clutter). Accuracy is implicitly tested as the perception component is a limiting factor in grasp success. The current (top-1) prediction accuracy is sufficiently high (roughly 96\%) that the experiments primarily test robustness, with processing speed being evaluated for the dynamic object experiments. A brief discussion of findings concludes each experimental section. Supplemental Material is found at Xu et al. (2020b).

\subsection{Static Grasping}

The static grasping experiment consists of grasping trials for individual objects. It is a baseline test for understanding expected performance in ideal situations. The test primarily evaluates accuracy of grasping candidate prediction, and secondly robustness to object variation. It mirrors the experiments in (Morrison et al. 2019; Chu et al. 2018).
8.1.1 Experimental Setup and Methodology. For comparison to published methods whose implementations are publicly available, the static grasping experiments consist of tests on three object sets, the Morrison set, the Chu set, and the adversarial set, each reported individually. The choice of the object sets permits comparison with the published singleobject pipeline methods. In particular experiments on the Morrison set and adversarial set compare with Morrison et al. (2019), and the Chu set compare with Chu et al. (2018).

An experiment consists of 10 grasp trials for each object in the chosen object set. For a given object set, a single trial begins with an object placed within the camera's view. The manipulation process involves planning a pick sequence, whereby the manipulator first moves to a location $15 \mathrm{~cm}$ higher than the chosen grasp (along the $z$-axis) and oriented downwards, then rotates its wrist to achieve the predicted grasp angle. Once in this pre-grasp pose, the manipulator lowers the gripper to the grasp location and closes. The gripper is lifted $15 \mathrm{~cm}$ back up to the pre-grasp pose. The GKNet grasp candidate selected is the one with the highest confidence score.

8.1.2 Analysis of Outcomes. Tables 7, 8, and 9 collect the recorded outcomes of the grasping tests. They also present the published results of the analogous trials for the corresponding baseline method (Morrison et al. 2019; Chu et al. 2018; Mahler et al. 2017). The 95\% confidence intervals (as binary variable) are also computed for each method in all three tables. In Chu et al. (2018), two criteria for grasp selection were explored, the top-1 confidence and the nearest-to-center approach. To match the GKNet selection criteria, the outcome for top-1 confidence is reported in Table 8 ( $86 \%$ vs $89 \%$ for nearest-to-center). GKNet has the highest success rate across the three experimental datasets, which indicates that the keypoint representation for grasp prediction may be an effective means to hypothesize candidate grasps for unknown objects. Looking at the adversarial set outcomes, GKNet achieves similar but slightly better performance than Mahler et al. (2017) whose approach was trained on the test objects, which indicates the capability of GKNet to generalize to novel objects with rich interior geometry. Additionaly, GKNet 
Table 7. Static Grasping experiments results with $95 \%$ confidence intervals for household set (Morrison)

\begin{tabular}{|l|c|l|c|}
\hline object & Accuracy & object & Accuracy \\
\hline cup & $9 / 10$ & ball & $10 / 10$ \\
\hline brush & $9 / 10$ & toothbrush & $9 / 10$ \\
\hline bear toy & $10 / 10$ & dice & $10 / 10$ \\
\hline tape & $9 / 10$ & duck toy & $10 / 10$ \\
\hline marker & $10 / 10$ & clamper & $8 / 10$ \\
\hline screwdriver & $10 / 10$ & cable & $10 / 10$ \\
\hline \hline GKNet & \multicolumn{2}{|c|}{$95.00 \pm 3.90$} \\
\hline \multicolumn{2}{|l|}{ Morrison et al. (2019) } & \multicolumn{2}{|c|}{$92.00 \pm 4.85$} \\
\hline
\end{tabular}

Table 8. Static Grasping experiments results with $95 \%$ confidence intervals for household set (Chu)

\begin{tabular}{|l|c|l|c|}
\hline object & Accuracy & object & Accuracy \\
\hline banana & $10 / 10$ & stapler & $9 / 10$ \\
\hline glasses & $10 / 10$ & spoon & $10 / 10$ \\
\hline ball & $10 / 10$ & bowl & $10 / 10$ \\
\hline tape & $9 / 10$ & scissors & $9 / 10$ \\
\hline screwdriver & $10 / 10$ & mouse & $9 / 10$ \\
\hline GKNet & \multicolumn{2}{|c|}{$96.00 \pm 3.84$} \\
\hline \multicolumn{2}{|l|}{ Chu et al. (2018) } & \multicolumn{2}{|c|}{$86.00 \pm 6.80$} \\
\hline
\end{tabular}

Table 9. Static Grasping experiments results with $95 \%$ confidence intervals for adversarial set

\begin{tabular}{|l|c|l|c|}
\hline object & Accuracy & object & Accuracy \\
\hline bar_clamp & $10 / 10$ & gearbox & $10 / 10$ \\
\hline part1 & $9 / 10$ & vase & $9 / 10$ \\
\hline pawn & $10 / 10$ & turbine_housing & $10 / 10$ \\
\hline part3 & $8 / 10$ & nozzle & $10 / 10$ \\
\hline \hline GKNet & \multicolumn{2}{|c|}{$95.00 \pm 4.78$} \\
\hline Morrison et al. (2019) & \multicolumn{2}{|c|}{$84.00 \pm 8.03$} \\
\hline Mahler et al. (2017) & \multicolumn{2}{|c|}{$93.00 \pm 5.59$} \\
\hline
\end{tabular}

outperforms Morrison et al. (2019) by $11 \%$ and nearly matches its own performance on the household objects. This parity in outcomes for household versus adversarial sets suggests that the keypoint representation is less affected by adversarial examples. The confidence intervals of GKNet across the three tests indicate that these performance outcomes have statistical support. GKNet outperforms Chu et al. (2018) for the Chu household set and Morrison et al. (2019) for the adversarial set. GKNet achieves similar performance as Mahler et al. (2017) for the adversarial set. GKNet and Morrison et al. (2019) performance is similar for the Morrison household set.

When compared to the vision grasp detection accuracy results from Tables 4 and 6 , the performance gap between the perception only and the perceive-plan-action pipelines is smallest for GKNet. There is a less than $1 \%$ difference in success rate from the vision-only experiment to the manipulation experiments. In contrast Chu et al. (2018) drops by $10 \%$, while Morrison et al. (2019) performs poorly for vision-only but executes well. The vision versus manipulation gap would suggest higher variation in grasp performance outcomes for Morrison et al. (2019) as more objects are tested.

8.1.3 Discussion. The results show that GKNet can generate accurate grasp predictions on isolated static objects that translate well to actual execution for an embodied robotic arm. It is capable of predicting reliable grasp candidates for objects with complex and adversarial
Table 10. Grasping Comparison to Published Works

\begin{tabular}{|l|c|c|c|c|}
\hline Approach & Year & \multicolumn{2}{|c|}{ Settings } & $\begin{array}{c}\text { Success } \\
\text { Rate (\%) }\end{array}$ \\
\hline & & Objects & Trials & \\
\hline Jiang et al. (2011) & 2011 & 12 & - & 87.9 \\
\hline Lenz et al. (2015) & 2015 & 30 & 100 & $84.0 / 89.0$ \\
\hline Pinto and Gupta (2016) & 2016 & 15 & 150 & 66.0 \\
\hline Johns et al. (2016) & 2016 & 20 & 100 & 80.3 \\
\hline Watson et al. (2017) & 2017 & 10 & - & 62.0 \\
\hline Mahler et al. (2017) & 2017 & 10 & 50 & 80.0 \\
\hline Asif et al. (2017) & 2017 & 35 & 134 & 91.9 \\
\hline Viereck et al. (2017) & 2017 & 10 & 40 & 97.5 \\
\hline Morrison et al. (2019) & 2018 & 20 & 200 & 87.0 \\
\hline Chu et al. (2018) & 2018 & 10 & 100 & 89.0 \\
\hline Satish et al. (2019) & 2019 & 8 & 80 & 87.5 \\
\hline Asif et al. (2019) & 2019 & - & $>200$ & $89.0 / 90.0$ \\
\hline Lu et al. (2020) & 2020 & 10 & 30 & 84.0 \\
\hline \hline GKNet & - & 30 & 300 & $95.3 \pm 2.4$ \\
\hline \hline Kopicki et al. (2016) & 2016 & 45 & 45 & 77.8 \\
\hline ten Pas and Platt (2018) & 2018 & 30 & 214 & 85.0 \\
\hline Liang et al. (2019) & 2019 & 10 & 100 & 82.0 \\
\hline Mousavian et al. (2019) & 2019 & 17 & 51 & 88.0 \\
\hline Lin et al. (2020) & 2020 & 10 & 100 & 94.0 \\
\hline Lou et al. (2020) & 2020 & 5 & 50 & 82.5 \\
\hline Wu et al. (2020) & 2020 & 20 & 60 & 85.0 \\
\hline
\end{tabular}

* Success rate of $84 \% / 89 \%$ achieved on Baxter / PR2 robot.

** Success rate of $89 \% / 90 \%$ achieved on ResNet/DenseNet.

shapes. The tests confirm that GKNet is robust to object shape. The consistency between vision-only and embodied manipulation grasp accuracy suggests that GKNet might have improved robustness properties over other RGB-D grasp identification pipelines. Additional evidence for this possibility lies in the next subsection, which compares grasping outcomes with other published works.

\subsection{Comparison of Grasping Outcomes with Published Work}

To place the performance of GKNet within the greater context of existing grasping approaches, Table 10 collects the success rates of published grasping research efforts. All results cited involved static grasping of single objects located on a tabletop workspace as measured by a single camera view, with the exception of Viereck et al. (2017) which employed eye-in-hand visual servoing. Since other experimental conditions in the published outcomes varied, we include in the table two details of the physical grasping experiments: the number of unique objects tested and the total number of trials across the objects. In a few cases, one or the other quantity was missing. The placement of GKNet in the table is intentional, as the algorithms above it process image data and output 2D grasp representations, while the algorithms below it use point cloud information and 3D grasp representations. The method in Lin et al. (2020) is a mixed method whose CNN segments the depth data to support grasp synthesis post-processing on point clouds.

8.2.1 Discussion of Source Data. Across the published works, the grasping approaches were tested with different object sets, numbers of total trials, and successful grasp criteria. Some of the differences are noted here. The majority of the listed works perform experiments on common 
household objects collected by the respective authors, thus there will be some differences in the object sets. However, more recent works tend to obtain their sets from existing benchmark datasets (Calli et al. 2015a; Leitner et al. 2017), or attempt to match those of others like (Mahler et al. 2017). In some cases the experiments were not repeated trials from a fixed set of objects so they will not divide (trials by objects) evenly. For evaluating success, almost all works utilize a similar policy: the grasp is successful when the object is grasped and lifted in the air. DexNet 2.0 Mahler et al. (2017) has a slightly different evaluation metric which requires the gripper to keep hold of the object after lifting, transporting, and shaking. One difference regarding the GKNet and Morrison et al. (2019) rows is that they aggregate the statistics from Tables 7-9, meaning that the success rate percentages include common household objects and adversarial objects. In spite of these differences, rough comparisons can be made since the experiments involved single objects on a clutter-free tabletop. Two publications reported grasping outcomes using multiple views, which are are not included in the table. Kopicki et al. (2016) achieved 84.4\% with 7 views and ten Pas and Platt (2018) improved the performance to $87.8 \%$ with a second depth camera for recovering a richer, more accurate point cloud.

8.2.2 Analysis of Outcomes Using the confidence interval of GKNet as a starting point for the comparison, only three methods score higher than the lower limit of the confidence interval (92.9\%), Viereck et al. (2017) and Lin et al. (2020). The remaining outcomes can be considered to be lower performing grasping methods presuming that the grasping tests are roughly similar. For certain object subsets, the methods may perform comparably, but for more general tasks they will most likely exhibit lower performance. Returning to Viereck et al. (2017), whose success rate is at the high end of the GKNet confidence interval. Two properties differentiate the results, with the first being the lower numbers of objects and trials per object, and the second being the eye-in-hand visual servoing algorithm design. The difference in trials lends some statistical uncertainty to the comparison, while the eye-in-hand nature makes the algorithm more compatible with the later test of dynamic eye-to-hand grasping in Sec. 8.4, where the performance gap is smaller; GKNet averages $96.5 \%$ for a $1 \%$ performance gap. The eye-in-hand approach permits multiple estimates from potentially different views over time and may reduce the likelihood of a grasp error based on how the sequential estimates are generated and processed. Additionally, the tests of different viewpoints, Sec. 8.3, suggest that there may be a view capable of consistently matching the performance of Viereck et al. (2017) from a single image. The best performing viewing angle scores $97 \%$ for a $0.5 \%$ performance gap. There is also value to exploring what additional, complementary implementation details from Viereck et al. (2017) might translate to GKNet: unlike GKNet, Viereck et al. (2017) is a grasp sampling and scoring mechanism. Thus, rather than elect the top- 1 grasp candidate, it might be useful to score and select from amongst the top candidates. The findings in Satish et al. (2019) suggests that this would be of value, though the outcomes of Satish et al. (2019) for individual object grasping do not reflect it.
Lastly for Lin et al. (2020), whose performance is just below that of GKNet, the grasping approach prioritizes the detection of shape primitives to inform grasp hypothesis generation and scoring. It consists of a deep learning primitive segmentation step followed by a more traditional, and slower, model-based grasping pipeline. The pipeline generates 3D grasp candidates and scores them to output a single grasp to execute. Given the strong performance of this system, there is a value to more explicitly encoding shape into the network structure or processing pipeline, followed by a GKNet form of grasp recognition (with grasp re-ranking).

8.2.3 Discussion. In summary, GKNet represents a top performing grasp pipeline with a different and complementary network and processing structure to comparably performing systems. GKNet's processing time is amongst the fastest of existing methods. The tests span a diverse set of objects, such that the high success rate demonstrates consistency of grasp performance relative to variation in object shape. This provides evidence for the robustness of GKNet to object shape or form, for graspable objects whose size is comparable to the gripper opening distance. The strength of GKNet lies in the keypoint representation, which should be studied further to understand how it may naturally integrate with explicit primitive shape modeling and grasp scoring methods, and how it may extend to $S E(3)$ grasps.

\subsection{Grasping at Varied Camera Angles}

The Cornell and AJD datasets are created from annotating imagery with similar camera views, effectively a top-down view. Consequently, training of GKNet uses imagery with a top-down camera perspective, which need not be the configured perspective for a manipulation setup. In such cases, it is important for the grasping algorithm to provide consistent grasping performance insensitive to deviations from the top-down perspective. The experiment described in this section evaluates static grasping performance consistency to varied camera angles.

8.3.1 Experimental Setup and Methodology. The setup and methodology is similar to the static grasping experiments, with camera viewing angle as the independent variable. The view angles vary from $90^{\circ}$ to $45^{\circ}$ in $15^{\circ}$ decrements such that the distance from the object to the camera frame is consistent across the experiments, see Fig. 10. The object set used is the compound object set, Fig. 9(d). A top-down grasp is commanded to pick each object. There are 10 grasp trials per object with randomized poses for each trial. The view angle does not go below $45^{\circ}$ because the data pre-processing (§5.1) approach is incompatible with learning to grasp on highly skewed background planes, as occurs for table-like support surfaces viewed at low incidence angles.

8.3.2 Analysis of Outcomes. As shown in Table 11, GKNet achieves grasp success rate of $95.00 \%, 97.00 \%$, $94.00 \%$ and $93.00 \%$ for the camera orientation of $90^{\circ}, 75^{\circ}$, $60^{\circ}$ and $45^{\circ}$, respectively. Among the results, the experiment of $75^{\circ}$ camera angle achieves the best outcome, while the outcome of $45^{\circ}$ camera angle is the worst. Interestingly, the $75^{\circ}$ success rate lies quite close to that of Viereck et al. 


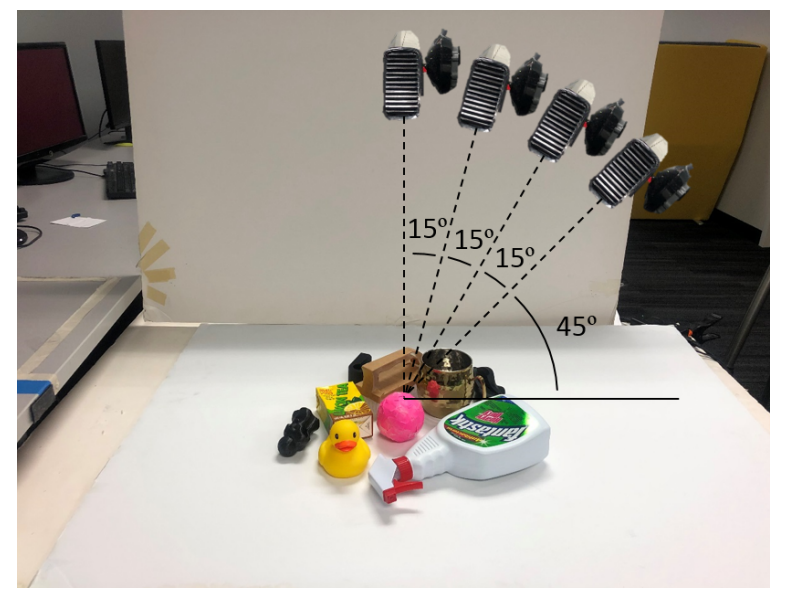

Figure 10. Experimental setup for grasping at varied camera angles (side view).

(2017) from Table 10, indicating that GKNet could possibly be configured to achieve state-of-the-art performance.

Choosing the outcome of $75^{\circ}$ as the reference, outcomes of $90^{\circ}$ and $60^{\circ}$ fall inside its $95 \%$ confidence interval (binary variable) while the outcome of $45^{\circ}$ falls just outside. The $45^{\circ}$ viewpoint sufficiently differs in image structure that network inference begins to degrade, albeit by a small amount as indicated by the fact that the $90^{\circ}, 60^{\circ}$, and $45^{\circ}$ outcomes are close to each other. Looking at Table 10, all of the views continue to outperform the majority of the other methods when considering the lower boundaries of the intervals. The approach whose outcome lies within the confidence intervals of the $90^{\circ}, 60^{\circ}$, and $45^{\circ}$ cases is Asif et al. (2017). For the $45^{\circ}$ case, three methods lie within the confidence interval: Asif et al. (2019); Chu et al. (2018); Lenz et al. (2015). In all cases, GKNet mean values are higher and the methods are at the lower end of the intervals. The $45^{\circ}$ viewpoint reflects a significant view change given that GKNet was designed under the assumption of consistent background depth values, which a $45^{\circ}$ viewing angle does not have. The difficulty is reflected in the lower success rate and larger confidence interval.

8.3.3 Discussion. These aggregate outcomes indicate that GKNet is relatively robust to camera viewing angle (up to $45^{\circ}$ as tested). It will work well for camera configurations that deviate from top-down views, which commonly happens when configuring a camera to operate as an eye-to-hand manipulation system. The lower performance at the more oblique angle $\left(45^{\circ}\right)$ may result from the loss of topdown geometry impacting top-down grasping. In these cases, approaching from an angle more consistent with the viewpoint should improve performance. More extreme camera angles $\left(30^{\circ}\right.$ or less) would benefit from estimation and isolation of the dominant plane to remove its impact, from generalizing grasps to not require top-down views, or from pursuing $S E(3)$ grasp recognition extensions.

\subsection{Dynamic Grasping}

An additional nuisance factor that may occur during manipulator deployment is object movement in a consistent direction, such as when grasping from a conveyor belt or other consistently moving support surface. A dynamic
Table 11. Results of grasping at varied camera angles experiments with $95 \%$ confidence intervals for the compound set.

\begin{tabular}{|l|c|c|c|c|}
\hline \multirow{2}{*}{ object } & \multicolumn{4}{|c|}{ Accuracy (\%) } \\
\cline { 2 - 5 } & $90^{\circ}$ & $75^{\circ}$ & $60^{\circ}$ & $45^{\circ}$ \\
\hline bar_clamp & $10 / 10$ & $10 / 10$ & $10 / 10$ & $10 / 10$ \\
\hline pawn & $10 / 10$ & $10 / 10$ & $9 / 10$ & $10 / 10$ \\
\hline vase & $8 / 10$ & $9 / 10$ & $9 / 10$ & $9 / 10$ \\
\hline tape & $10 / 10$ & $10 / 10$ & $10 / 10$ & $10 / 10$ \\
\hline sprayer & $10 / 10$ & $10 / 10$ & $9 / 10$ & $9 / 10$ \\
\hline juice box & $10 / 10$ & $10 / 10$ & $10 / 10$ & $10 / 10$ \\
\hline duck toy & $9 / 10$ & $10 / 10$ & $9 / 10$ & $9 / 10$ \\
\hline cup & $9 / 10$ & $9 / 10$ & $9 / 10$ & $8 / 10$ \\
\hline brush & $10 / 10$ & $9 / 10$ & $10 / 10$ & $10 / 10$ \\
\hline ball & $9 / 10$ & $10 / 10$ & $9 / 10$ & $8 / 10$ \\
\hline \hline average (\%) & 95.00 & 97.00 & 94.00 & 93.00 \\
95\% interval & \pm 4.27 & \pm 3.34 & \pm 2.37 & \pm 5.00 \\
\hline
\end{tabular}

grasping experiment following the protocol of Morrison et al. (2019) tests real-time robustness to object movement.

8.4.1 Implementation. To evaluate GKNet in real-time tasks, we perform dynamic grasping by implementing a Position Based Visual Servoing (PBVS) controller (Kragic and Christensen 2002). PBVS control requires real-time tracking of the robot pose to compute the error between the current and desired pose. For the eye-to-hand camera view of the grasping setup, the Depth-Based Robot Tracking (DBRT) algorithm (Cifuentes et al. 2016) estimates the current pose of the robot from a depth camera stream. The depth image stream is generated at $30 \mathrm{~Hz}$ by a Kinect Xbox 360 . A simple detection-based tracking method informs grasp selection. At the begining of each round, GKNet outputs the grasp detection with the highest confidence score and the robot is controlled by the PBVS controller to move accordingly. Since the object is expected to move, future processing from GKNet extracts the top 5 grasp candidates for each frame and compares these five predictions with the selected one at the previous frame. The grasp candidate with the lowest Euclidean distance to the previous grasp selection is chosen as the grasp to achieve, if it is below a tolerance threshold, $\tau_{\text {close. }}$ If no grasp passes the threshold, then the previous grasp pose remains the target grasp pose, under the presumption that the gripper has occluded the object. Closed-loop control will be stopped after the robot reaches the predicted position and is about to grasp the object. Upon reaching the target pose, the gripper closes to grab the object, then lifts.

8.4.2 Experimental Setup and Methodology. The setup and methodology is similar to the static grasping experiments, however the target object is moved by the experimenter with a translation of at least $100 \mathrm{~mm}$ and a rotation of at least $25^{\circ}$ about the object's center during each individual trial. The direction of travel is linear and randomly chosen, as is the rotation, and is effected by moving a piece of paper upon which the object is placed. The movement is a slow continuous action beginning right after the manipulator starts to move. The entire movement lasts for 3 seconds. There are 10 grasp trials for each object in the household set (Morrison) and the adversarial set, for 200 grasp trials in total. The grasp attempt is rated successful if the object is lifted. 
Table 12. Results of dynamic grasping experiments with $95 \%$ confidence intervals for household set (Morrison)

\begin{tabular}{|l|c|l|c|}
\hline object & Accuracy & object & Accuracy \\
\hline cup & $10 / 10$ & ball & $9 / 10$ \\
\hline brush & $9 / 10$ & toothbrush & $10 / 10$ \\
\hline bear toy & $10 / 10$ & dice & $10 / 10$ \\
\hline tape & $9 / 10$ & duck toy & $10 / 10$ \\
\hline marker & $10 / 10$ & clamper & $9 / 10$ \\
\hline screwdriver & $10 / 10$ & cable & $10 / 10$ \\
\hline \hline GKNet & \multicolumn{2}{|c|}{$96.67 \pm 3.21$} \\
\hline Morrison et al. (2019) & \multicolumn{2}{|c|}{$88.00 \pm 5.81$} \\
\hline
\end{tabular}

Table 13. Results of dynamic grasping with $95 \%$ confidence intervals for adversarial set

\begin{tabular}{|l|c|l|c|}
\hline object & Accuracy & object & Accuracy \\
\hline bar_clamp & $10 / 10$ & gearbox & $10 / 10$ \\
\hline part1 & $8 / 10$ & vase & $9 / 10$ \\
\hline pawn & $10 / 10$ & turbine_housing & $10 / 10$ \\
\hline part3 & $10 / 10$ & nozzle & $10 / 10$ \\
\hline \hline GKNet & \multicolumn{2}{|c|}{$96.25 \pm 4.16$} \\
\hline Morrison et al. (2019) & \multicolumn{2}{|c|}{$83.00 \pm 8.23$} \\
\hline
\end{tabular}

8.4.3 Analysis of Outcomes. Tables 12 and 13 report the individual trial outcomes for the object and the success rate. GKNet achieves $96.67 \%$ and $96.25 \%$ on the household set (Morrison) and adversarial set. These success rates outperform Morrison et al. (2019) by $8.67 \%$ and $13.25 \%$, which is greater than GKNet's performance advantage in the static grasping experiments. Meanwhile the outcomes of Morrison et al. (2019) fall below and outside of the GKNet's confidence intervals (binary variable) providing additional support that GKNet outperforms the baseline.

8.4.4 Discussion. Qualitatively, the outcomes demonstrate that GKNet processing speed and grasp candidate accuracy are both sufficiently good to permit grasping of slowly continuously moving objects, when combined with a compatible tracking strategy. Quantitatively, the similar average and confidence intervals of the dynamic grasping success rates to those of the static grasping success rates confirms robustness of grasp candidate generation to movement. GKNet's competitive and consistent performance versus the baseline provides further evidence that a keypoint approach to grasp recognition is more beneficial relative to other representations for parallel-plate type grippers, or grippers functionally similar to them.

\subsection{Bin Picking}

The last experiment to perform is a bin picking experiment similar to Mahler and Goldberg (2017) and Morrison et al. (2019). The experiment evaluates robustness of grasp recognition to environmental clutter, which would thereby examine the generalization capabilities of GKNet since the network is trained with images containing only individual objects. Multi-object and cluttered environments are scenarios anticipated to be experienced for some realworld deployments. Thus providing reliable grasp prediction to other sensed objects is an essential capability of grasp detection approaches.

8.5.1 Implementation. As a grasp candidate generation strategy, GKNet does not provide a means to identify which candidate grasp is actually the best to execute in cluttered settings. Grasping objects in cluttered scenes usually involves grasp scoring algorithms that take into consideration potential collisions and occlusion geometry (Fischinger and Vincze 2012; Fischinger et al. 2013; Domae et al. 2014; ten Pas et al. 2017). Unlike deep learning grasp quality scoring methods specifically trained for this problem (Mahler and Goldberg 2017), GKNet requires additional post-processing to operate in clutter. The additional postprocessing is a grasp quality scoring function designed based on geometric and heuristic approaches (Gualtieri et al. 2016; ten Pas et al. 2017). The grasp scoring algorithm implemented for this experiment takes a single top-down view depth image and a set of grasp detections as input, then outputs scores for each grasp detection with respect to collision, occupancy, and grasp height, as described below.

The collision score evaluates the potential for collision by the two "fingers" at the chosen grasp pose. Given the topdown view depth image, scoring will involve a simplified model of the 3D gripper into a 2D projected model as shown in Fig. 11 (a). Two projected red rectangles represent the two sides of gripper, with a third blue rectangle representing the open area within the two fingers. For collision checking, per Fig. 11 (b), the algorithm processes the pixels inside the rectangles and computes the ratio of pixels whose depth is larger than the depth of the center keypoint. Denote the union of pixel elements in the two rectangular regions by $\mathcal{G}$, then the collision score is

$$
s_{c}=\frac{1}{|\mathcal{G}|} \sum_{p \in \mathcal{G}} \mathrm{H}\left(d(p)-d\left(p_{c}\right)\right),
$$

where $\mathrm{H}(\cdot)$ is the Heaviside function, $p_{c}$ is the center keypoint, and $d(\cdot)$ is the depth. The collision score ranges between 1 (no collisions) to 0 (all collisions).

The occupancy score computes the number of pixels whose depth is higher the support surface. For occupancy checking, per Fig. 11 (c), the main region of interest lies within the grippers where a hypothesized object part to grasp lies. Denote this gripper interior region by $\mathcal{O}$. The occupancy score compares the depth values to the nominal depth of the table or supporting surface at the center keypoint,

$$
s_{o}=\frac{1}{|\mathcal{O}|} \sum_{p \in \mathcal{O}} \mathrm{H}\left(d_{S}\left(p_{c}\right)-d(p)\right)
$$

where $d_{S}(\cdot)$ is the depth map for the supporting surface. The occupancy score varies from 0 to 1 .

The last score involved in the composite grasp scoring function is the grasp height score, which prioritizes grasping objects from the top of the heap. It is computed as the normalized vertical height off the surface for the hypothesized object located at the center keypoint:

$$
s_{h}=\frac{\left|d\left(p_{c}\right)-d_{S}\left(p_{c}\right)\right|}{\left|d_{S}\left(p_{c}\right)\right|} .
$$

Its range is between 0 and 1 , so that the outputs of all three scores lie within the same range. The final grasp score is the sum of the scores, $s_{g}=s_{c}+s_{o}+s_{h}$.

8.5.2 Experimental Setup and Methodology. The setup of the bin picking experiment is shown in Fig. 12. The experiment itself follows the protocol in (Mahler and 

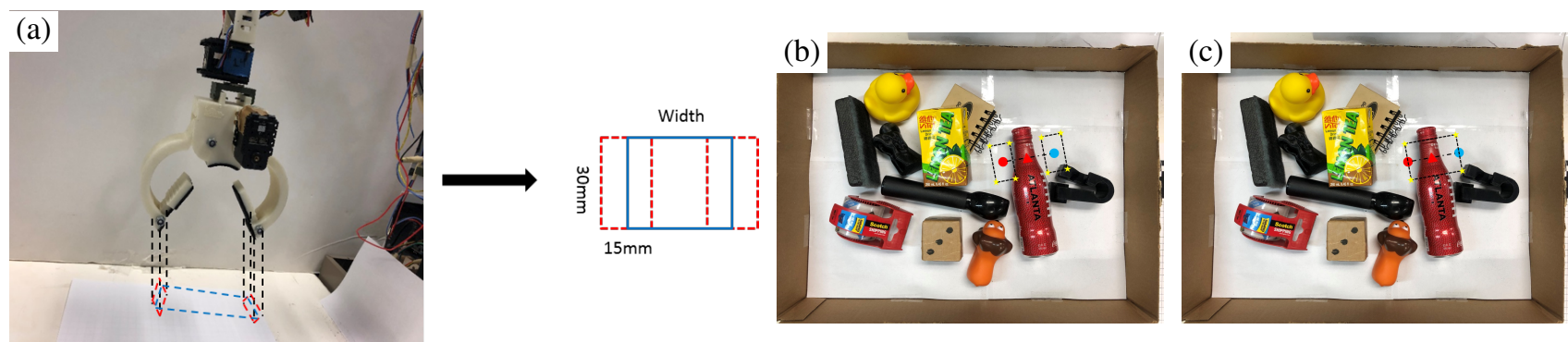

Figure 11. (a) Simplified 2D gripper model. Red dotted boxes represent the two sides of gripper and blue solid box represents the area within the gripper. The gripper model informs the grasp scoring contributions for (b) collisions, and (c) occupancy.

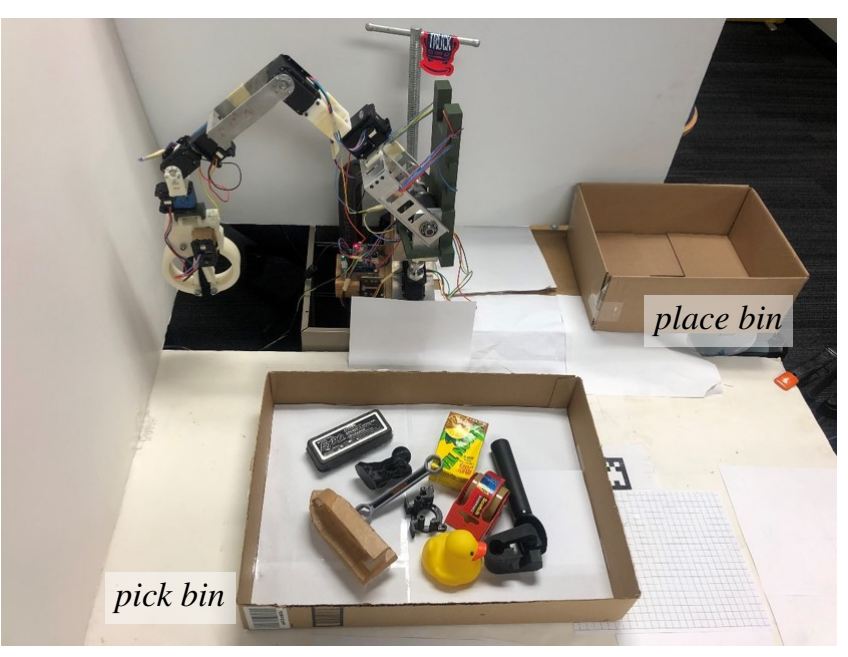

Figure 12. Setup of the bin picking experiment. Objects placed into the pick bin should be moved to the place bin

Goldberg 2017; Morrison et al. 2019), and is outlined in Algorithm 2. The grasp attempt of an object in the pick bin is labeled successful if the robot pick and place action leads to the object being placed in the place bin. The entire experiment iterates until (a) there are no objects left in the bin or (b) the robot fails to grasp the same object 5 consecutive times. The bin picking object set, Fig. 9 (e), is used.

Trial Configurations and Evaluation. The bin picking experiment is performed under two different configurations. In one, the number of objects is $N=5$ and the number of trials is 20. In the second, the number of objects is $N=10$ and the number of trials is 10 . In addition to the grasp success rate (SR), a second evaluation metric is the percent cleared (PC). The percent cleared is the fraction of objects that were moved to the place bin. Confident intervals will treat the SR and $\mathrm{PC}$ values as continuous variables.

Baseline Methods and Comparative Evaluation. The three comparative baseline methods and scores come from Mahler and Goldberg (2017). They are an image-based Force Closure method, and the DexNet 2.0 and DexNet $2.1(\epsilon=$ $0.9)$ methods. The Force Closure method performs a random planar force grasp with friction coefficient $\mu=0.8$ computed from edge detection in depth images. It also employs a model-based grasp scoring system based on (ten Pas and Platt 2018; Gualtieri et al. 2016). Dex-Net 2.0 employs the GQ-CNN model to rank grasps, while Dex-Net 2.1 ranks grasps using the Dex-Net 2.1 classifier.

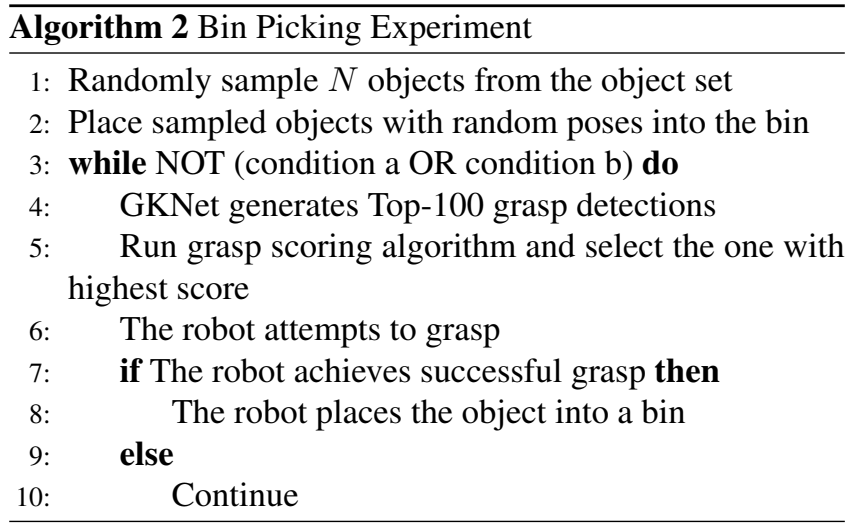

The experimental implementation in Mahler and Goldberg (2017) is not exactly the same as the one performed here. There are two main experiments that are closest to the current ones. They are labelled (S1) and (S2), based on the object set. The object set (S1) consists of 25 opaque and rigid objects, where the number of trials was set to 20 and 10 for $N=5$ and $N=10$, respectively. The second object set (S2) consists of 50 objects, with the additional 25 being transparent, hinged, and deformable objects. There were 5 trials per object.

The bin picking object set used here is similar to the set with full 50 objects used in Mahler and Goldberg (2017) per their description, but lying between (S1) and (S2) in difficulty. Results from (S1) and (S2) thus establish nominal upper and lower bounds that would be considered good if the performance of GKNet with simple grasp quality scoring were to lie near to or within them. Force Closure has an established gap versus learning methods (Mahler and Goldberg 2017). GKNet should perform better than the Force Closure method since it combines a learning-based grasp hypothesis strategy with model-based grasp scoring. In essence, it replaces the randomized grasp sampling.

8.5.3 Analysis of Outcomes. Table 14 collects the outcomes of the bin picking experiment. For the 5 object setting, the 20 trials led to a $92.1 \%$ grasp success rate and $100 \%$ of objects cleared. For the 10 object setting, the 10 trials led to a $72.1 \%$ grasp success rate and $98 \%$ of objects cleared. Reviewing the results relative to the baselines indicates that GKNet plus simple grasp quality scoring does indeed have the desired properties described in Section 8.5.2. For the $N=5$ case it outperforms Force Closure (S1) and nearly matches Dex-Net 2.0 (S1) and Dex-Net 2.1 (S1). Recall that (S1) is qualitatively an easier set relative to the 
Table 14. Bin Picking Experiment

\begin{tabular}{|c|c|c|c|c|}
\hline \multirow{2}{*}{ Approach } & \multicolumn{2}{|c|}{$N=5$} & \multicolumn{2}{|c|}{$\mathbf{N}=\mathbf{1 0}$} \\
\hline & $\mathbf{S R}^{*}$ & $\mathbf{P C}^{* *}$ & SR & PC \\
\hline GKNet & 92.1 & 100.0 & 72.1 & 98.0 \\
\hline Force Closure (S1) & 54.0 & 97.0 & 55.0 & 92.0 \\
\hline Dex-Net $2.0(\mathrm{~S} 1)$ & 92.0 & 100.0 & 83.0 & 98.0 \\
\hline Dex-Net $2.1(\mathrm{~S} 1)$ & 94.0 & 100.0 & 89.0 & 100.0 \\
\hline Force Closure (S2) & - & - & 50.0 & 77.0 \\
\hline Dex-Net 2.0 (S2) & - & - & 81.0 & 98.0 \\
\hline Dex-Net 2.1 (S2) & - & - & 85.0 & 100.0 \\
\hline
\end{tabular}

bin picking set, and GKNet is trained only with individual objects, thus one would anticipate higher performance for Dex-Net variants. The $95 \%$ confidence interval for GKNet is $92.1 \pm 4.8$, which is above the Force Closure and contains the DexNet variants.The interval supports the conclusions that GKNet outperforms Force Closure and is comparable to the DexNet variants for this scenario. The comparable performance assessment is further supported by the $100 \%$ bin-picking percent cleared rates for GKNet and the DexNet variants. For the $N=10$ case there is a larger difference for the methods, with GKNet performing between Force Closure and the DexNet variants in terms of success rate. The percent cleared matches the Dex-Net 2.0 (S1) and (S2) outcomes. The $95 \%$ confidence interval of GKNet is $98.0 \pm 2.9$.

For the set (S2) and the $N=10$ case, the GKNet solution continues to outperform Force Closure with a percent cleared performance close to, but below, the DexNet variants. The success rate lies between Force Closure and the DexNet variants, but closer to the DexNet variants than to Force Closure. The confidence interval of $72.1 \pm 5.4$ continues to support the conclusion that GKNet improves upon Force Closure, but in this scenario does not match the performance of the Dex-Net variants. As with (S1), however, the percentage of bin-picking trials cleared again suggests comparable performance to the DexNet 2.0 variant for the task. Since Dex-Net is a grasp scoring CNN trained explicitly to handle cluttered scenes, it should have an advantage over model-based grasp quality scoring systems. The recent effort (Satish et al. 2019) suggests that combining convolutional network predictions with Dex-Net would give strong performance, since one limitation of Dex-Net is the sampling step. Future work should explore the performance of bin picking using a combined GKNet + Dex-Net method.

The failure cases split into visual and execution errors. On the visual side, the employed scoring algorithm prioritizes grasps whose fingers have less chance to collide with other objects. In cluttered environments such grasps may be at the corners or edges of objects, which do not lead to reliable grasp closure. Secondly, GKNet sometimes treats multiple objects as one entity and provide grasp keypoint pairings that spans them, as though trying to grab multiple objects at once. In the end, the manipulator grabs none of them. Most execution failures were caused by the design of the gripper. The gripper on the manipulator is not a true parallel plate gripper, but is a jaw-like gripper. The volume of space traversed during grasp closing is a bit larger than the true parallel-plate gripper. The simple grasp quality scoring system does not factor this design detail, which becomes a more critical factor as the clutter level increases. Employing an imitation learning or reinforcement learning grasp quality scoring system like Dex-Net for handling clutter should address this hard to model aspect. There were unique errors for the $N=10$ experiment that prevented full clearance of the objects. GKNet plus simple grasp quality scoring has problems selecting accurate grasp estimates for the small ketchup container, top-middle of Fig. 9(e), and for the blue plush bear, just below the ketchup in Fig. 9(e). Of all the grasp candidates, it would select the same failed grasp candidate, such that a single failure would necessarily imply 5 sequential failures. These two objects are the major source of performance degradation. Providing a means to recognize failed grasp attempts and choose alternative grasp candidates would be one way to correct the sequential mistakes.

8.5.4 Discussion. The GKNet bin-picking results show that GKNet is good at hypothesizing grasp candidates, while its use with a model-based grasp scoring and prioritization system could match learning based methods (i.e., Dex-Net grasp quality scoring) for low clutter picking scenarios. There was some degradation in success rate for more cluttered scenarios, which impacted the clearance rate. Further study is needed to understand how to bridge the light clutter to heavy clutter gap. Incorporating grasp quality neural network (GQ-CNN) designs as the grasp scoring mechanism should be one means to do so, especially if the GQ-CNN models were trained with diverse clutter scenarios.

8.5.5 Comparison to 6-DoF Grasping Methods. Grasping objects in clutter is a commonly conducted experiment for 6 DoF grasping methods (Murali et al. 2020; Sundermeyer et al. 2021; ten Pas et al. 2017; Lou et al. 2020; Liang et al. 2019; Ni et al. 2020; Zhao et al. 2020; Qin et al. 2020; ten Pas and Platt 2018). The goal of clutter removal is to move the objects somewhere else, which is similar to bin picking. Across these published works, experiments were conducted under similar protocols but can differ in the object set, number of objects placed in the scene, number of total runs, and stopping criteria of each run. Therefore, these results are not put into Table 14 but discussed here (see the Supplementary Material for the data in table form).

Lou et al. (2020) used toy blocks as the object set while the other works tested with common household objects. Most tests placed 4-10 objects on the table except Lou et al. (2020), which placed 30 blocks. There are mainly three types of stopping criteria among these works. Murali et al. (2020); Sundermeyer et al. (2021) limited grasp attempts to the number of objects in the scene, ten Pas et al. (2017); Lou et al. (2020); ten Pas and Platt (2018) chose to terminate when the table had been cleared or a given number of consecutive failures occurred for the same object, while Liang et al. (2019); Ni et al. (2020); Zhao et al. (2020); Qin et al. (2020) performed a fixed number of attempts greater than the number of objects in the scene. Most works reported both success rate and percent cleared, except (Murali et al. 2020; Sundermeyer et al. 2021); these latter two will not be compared due to a different methodology. In spite of these differences, rough comparisons can be made.

For success rate, the highest reported one is $93 \%$ from (ten Pas et al. 2017), which was conducted with multiple camera views; the SR dropped to $84 \%$ with two views. 
The best single-view method (Liang et al. 2019) reported an $89.33 \%$ success rate on an easy object set and $66.2 \%$ for a harder object set ( $N=8$ in both cases). The rest of the outcomes fall into the range of $71.43 \%$ to $82.95 \%$ and have $N \in\{6,10\}$. The GKNet success rate for the easy case $(92.1 \%)$ lies above the best performing methods, while for the hard case $(72.1 \%)$ it lies at the lower end of the range for all methods. For percent cleared, Lou et al. (2020) achieved $100 \%$, and Liang et al. (2019) achieved $100 \%$ (easy) and $95 \%$ (harder), while the rest of the works reported outcomes between $85 \%$ to $97.5 \%$. GKNet's percent clearance $(100 \%$ and $98 \%$ ) is close to the top performers. Overall, GKNet's performance is comparable to that of $6-\mathrm{DoF}$ methods in spite of considering a more limited 4-DoF grasp space (note: DexNet variants are top performers). The results indicate that the full dimensional grasp space can be downgraded to a topdown subspace for some well-structured industrial scenarios, e.g., bin picking tasks. The three 6-DoF methods achieving the best success rate or percent cleared reflect deep network grasp scoring methods using sampled grasp poses. This fact again reinforces the idea that GKNet would benefit from the incorporation of a robust grasp scoring method to re-rank the candidate grasp set it outputs.

\section{Conclusion}

The grasp keypoint network (GKNet) poses grasp identification as a paired keypoint estimation problem facilitated by heatmaps within the network structure. The design choice is based on the hypothesis that: the paired keypoint representation, with dense output and fewer internal constraints to enforce, should provide strong performance for the task of grasp recognition. Secondly, by design, the keypoint representation balances the speed vs success tradeoff. Vision-only and actual robotics experiments verified the hypothesis and explored the overall robustness of GKNet to various nuisance factors. Given that GKNet's performance is only valuable if it can translate to the embodied case, the physical grasping experiments quantified and confirmed GKNet's accuracy, speed, and robustness. Here robustness has been evaluated with respect to object diversity, camera viewpoint, object movement, and clutter. Across the first three nuisance classes, GKNet has consistent performance and does not experience the variation in success rates of other methods. Performance was also consistent for light clutter but degraded for heavy clutter.

Roughly speaking, object grasping requires resolving the what, where, and how. GKNet targets the how part. Parallel work exploring the where question focusing on primitive shapes (Lin et al. 2020) achieves a high success rate but slow processing time; on the order of seconds due to the use of classical shape fitting and grasping ranking algorithms. Future work aims to streamline the process by merging the two conceptual approaches to grasping. Likewise, identifying a means to translate the 2D grasp representation to a full $3 \mathrm{D}$ grasp pose would remove the need for a top-down grasp and permit richer manipulation from more varied viewpoints. Recent work on affordances and keypoints (Xu et al. 2021) indicates that keypoints should work well for recovering $S E(3)$ grasp frames. Lastly, introducing grasp quality neural networks (Mahler et al.
2017; Morrison et al. 2019) would further resolve the what question for cluttered scenarios. To promote reproduction of the work, all code and data is open source (Xu et al. 2020b).

\section{Funding}

This work supported in part by the National Science Foundation under Award \#1605228. Any opinions, findings, and conclusions or recommendations expressed in this material are those of the author(s) and do not necessarily reflect the views of the National Science Foundation.

\section{References}

Asif U, Bennamoun M and Sohel FA (2017) RGB-D object recognition and grasp detection using hierarchical cascaded forests. IEEE Transactions on Robotics 33(3): 547-564.

Asif U, Tang J and Harrer S (2019) Densely Supervised Grasp Detector (DSGD). In: Proceedings of the AAAI Conference on Artificial Intelligence. pp. 8085-8093.

Bicchi A and Kumar V (2000) Robotic grasping and contact: A review. In: IEEE International Conference on Robotics and Automation. pp. 348-353.

Bohg J and Kragic D (2010) Learning grasping points with shape context. Robotics and Autonomous Systems 58(4): 362-377.

Calandra R, Owens A, Jayaraman D, Lin J, Yuan W, Malik J, Adelson EH and Levine S (2018) More than a feeling: Learning to grasp and regrasp using vision and touch. IEEE Robotics and Automation Letters 3(4): 3300-3307.

Calli B, Singh A, Walsman A, Srinivasa S, Abbeel P and Dollar AM (2015a) The YCB object and model set: Towards common benchmarks for manipulation research. In: IEEE International Conference on Advanced Robotics. pp. 510-517.

Calli B, Walsman A, Singh A, Srinivasa S, Abbeel P and Dollar AM (2015b) Benchmarking in manipulation research: The YCB object and model set and benchmarking protocols. arXiv preprint arXiv: 1502.03143 .

Chang AX, Funkhouser T, Guibas L, Hanrahan P, Huang Q, Li Z, Savarese S, Savva M, Song S, Su H, Xiao J, Yi L and Yu F (2015) ShapeNet: An information-rich 3d model repository. arXiv preprint arXiv:1512.03012

Chu FJ, Xu R and Vela PA (2018) Real-world multiobject, multigrasp detection. IEEE Robotics and Automation Letters 3(4): 3355-3362.

Cifuentes CG, Issac J, Wüthrich $\mathrm{M}$, Schaal $\mathrm{S}$ and Bohg $\mathrm{J}$ (2016) Probabilistic articulated real-time tracking for robot manipulation. IEEE Robotics and Automation Letters 2(2): 577-584.

Coelho J, Piater J and Grupen R (2001) Developing haptic and visual perceptual categories for reaching and grasping with a humanoid robot. Robotics and Autonomous Systems 37(2-3): 195-218.

Deng J, Dong W, Socher R, Li LJ, Li K and Fei-Fei L (2009) ImageNet: A large-scale hierarchical image database. In: IEEE Conference on Computer Vision and Pattern Recognition. pp. 248-255.

Depierre A, Dellandréa E and Chen L (2018) Jacquard: A large scale dataset for robotic grasp detection. In: IEEE/RSJ International Conference on Intelligent Robots and Systems. pp. 3511-3516. 
Domae Y, Okuda H, Taguchi Y, Sumi K and Hirai T (2014) Fast graspability evaluation on single depth maps for bin picking with general grippers. In: IEEE International Conference on Robotics and Automation. pp. 1997-2004.

Duan K, Bai S, Xie L, Qi H, Huang Q and Tian Q (2019) CenterNet: Keypoint triplets for object detection. In: IEEE International Conference on Computer Vision. pp. 6569-6578.

Ekvall S and Kragic D (2007) Learning and evaluation of the approach vector for automatic grasp generation and planning. In: IEEE International Conference on Robotics and Automation. pp. 4715-4720.

Eppner C, Höfer S, Jonschkowski R, Martín-Martín R, Sieverling A, Wall V and Brock O (2016) Lessons from the Amazon picking challenge: Four aspects of building robotic systems. In: Robotics: Science and Systems.

Fischinger D and Vincze M (2012) Empty the basket-A shape based learning approach for grasping piles of unknown objects. In: IEEE/RSJ International Conference on Intelligent Robots and Systems. pp. 2051-2057.

Fischinger D, Vincze M and Jiang Y (2013) Learning grasps for unknown objects in cluttered scenes. In: IEEE International Conference on Robotics and Automation. pp. 609-616.

Fujita M, Domae Y, Noda A, Garcia Ricardez G, Nagatani T, Zeng A, Song S, Rodriguez A, Causo A, Chen IM and Ogasawara $\mathrm{T}$ (2020) What are the important technologies for bin picking? Technology analysis of robots in competitions based on a set of performance metrics. Advanced Robotics 34(7-8): 560-574.

Gualtieri M, Ten Pas A, Saenko K and Platt R (2016) High precision grasp pose detection in dense clutter. In: IEEE/RSJ International Conference on Intelligent Robots and Systems. pp. 598-605.

Guo D, Sun F, Liu H, Kong T, Fang B and Xi N (2017) A hybrid deep architecture for robotic grasp detection. In: IEEE International Conference on Robotics and Automation. pp. 1609-1614.

He K, Zhang X, Ren S and Sun J (2016) Deep residual learning for image recognition. In: IEEE Conference on Computer Vision and Pattern Recognition. pp. 770-778.

Hsiao K and Lozano-Perez T (2006) Imitation learning of wholebody grasps. In: IEEE/RSJ International Conference on Intelligent Robots and Systems. pp. 5657-5662.

Huebner K and Kragic D (2008) Selection of robot pre-grasps using box-based shape approximation. In: IEEE/RSJ International Conference on Intelligent Robots and Systems. pp. 1765-1770.

Jeng KY, Liu YC, Liu ZY, Wang JW, Chang YL, Su HT and Hsu W (2020) Gdn: A coarse-to-fine (c2f) representation for endto-end 6-dof grasp detection. arXiv preprint arXiv:2010.10695

Jiang Y, Moseson S and Saxena A (2011) Efficient grasping from RGBD images: Learning using a new rectangle representation. In: IEEE International Conference on Robotics and Automation. pp. 3304-3311.

Johns E, Leutenegger S and Davison AJ (2016) Deep learning a grasp function for grasping under gripper pose uncertainty. In: IEEE/RSJ International Conference on Intelligent Robots and Systems. pp. 4461-4468.

Kamon I, Flash T and Edelman S (1996) Learning to grasp using visual information. In: IEEE International Conference on Robotics and Automation. pp. 2470-2476.
Kappler D, Bohg J and Schaal S (2015) Leveraging big data for grasp planning. In: IEEE International Conference on Robotics and Automation. pp. 4304-4311.

Kingma DP and Ba J (2015) Adam: A method for stochastic optimization. In: International Conference on Learning Representations.

Kopicki M, Detry R, Adjigble M, Stolkin R, Leonardis A and Wyatt JL (2016) One-shot learning and generation of dexterous grasps for novel objects. The International Journal of Robotics Research 35(8): 959-976.

Kragic D and Christensen HI (2002) Survey on visual servoing for manipulation. Computational Vision and Active Perception Laboratory, Fiskartorpsv 15: 2002.

Kumra S and Kanan C (2017) Robotic grasp detection using deep convolutional neural networks. In: IEEE/RSJ International Conference on Intelligent Robots and Systems. pp. 769-776.

Lab RL (2013) Cornell grasping dataset. http://pr.cs. cornell.edu/grasping/rect_data/data.php. Accessed: 2017-09-01.

Law H and Deng J (2018) CornerNet: Detecting objects as paired keypoints. In: European Conference on Computer Vision. Springer, pp. 734-750.

Le QV, Kamm D, Kara AF and Ng AY (2010) Learning to grasp objects with multiple contact points. In: IEEE International Conference on Robotics and Automation. pp. 5062-5069.

Leitner J, Tow AW, Sünderhauf N, Dean JE, Durham JW, Cooper M, Eich M, Lehnert C, Mangels R, McCool C, Kujala PT, Nicholson L, Pham T, Sergeant J, Wu L, Zhang F, Upcroft B and Corke P (2017) The ACRV picking benchmark: A robotic shelf picking benchmark to foster reproducible research. In: IEEE International Conference on Robotics and Automation. pp. 4705-4712.

Lenz I, Lee H and Saxena A (2015) Deep learning for detecting robotic grasps. The International Journal of Robotics Research 34(4-5): 705-724.

Levine S, Finn C, Darrell T and Abbeel P (2016a) End-to-end training of deep visuomotor policies. The Journal of Machine Learning Research 17(1): 1334-1373.

Levine S, Pastor P, Krizhevsky A and Quillen D (2016b) Learning hand-eye coordination for robotic grasping with large-scale data collection. In: International Symposium on Experimental Robotics. pp. 173-184.

Liang H, Ma X, Li S, Görner M, Tang S, Fang B, Sun F and Zhang J (2019) PointNetGPD: Detecting grasp configurations from point sets. In: IEEE International Conference on Robotics and Automation. pp. 3629-3635.

Lin Y, Tang C, Chu FJ and Vela PA (2020) Using synthetic data and deep networks to recognize primitive shapes for object grasping. In: IEEE International Conference on Robotics and Automation.

Lou X, Yang Y and Choi C (2020) Learning to generate 6-dof grasp poses with reachability awareness. In: International Conference on Robotics and Automation. IEEE, pp. 15321538.

Lu Q, Chenna K, Sundaralingam B and Hermans T (2020) Planning multi-fingered grasps as probabilistic inference in a learned deep network. In: Robotics Research. Springer, pp. 455-472.

Mahler J and Goldberg K (2017) Learning deep policies for robot bin picking by simulating robust grasping sequences. In: Conference on Robot Learning. pp. 515-524. 
Mahler J, Liang J, Niyaz S, Laskey M, Doan R, Liu X, Ojea JA and Goldberg K (2017) Dex-Net 2.0: Deep learning to plan robust grasps with synthetic point clouds and analytic grasp metrics. In: Robotics: Science and Systems.

Mahler J, Matl M, Liu X, Li A, Gealy D and Goldberg K (2018) Dex-Net 3.0: Computing robust vacuum suction grasp targets in point clouds using a new analytic model and deep learning. In: IEEE International Conference on Robotics and Automation. pp. 1-8.

Mahler J, Matl M, Satish V, Danielczuk M, DeRose B, McKinley S and Goldberg K (2019) Learning ambidextrous robot grasping policies. Science Robotics 4(26): eaau4984.

Mahler J, Pokorny FT, Hou B, Roderick M, Laskey M, Aubry M, Kohlhoff K, Kröger T, Kuffner J and Goldberg K (2016) DexNet 1.0: A cloud-based network of $3 \mathrm{~d}$ objects for robust grasp planning using a multi-armed bandit model with correlated rewards. In: IEEE International Conference on Robotics and Automation. pp. 1957-1964.

Morrison D, Corke P and Leitner J (2019) Learning robust, realtime, reactive robotic grasping. The International Journal of Robotics Research : 0278364919859066.

Mousavian A, Eppner C and Fox D (2019) 6-dof graspnet: Variational grasp generation for object manipulation. In: IEEE International Conference on Computer Vision. pp. 2901-2910.

Murali A, Mousavian A, Eppner C, Paxton C and Fox D (2020) 6dof grasping for target-driven object manipulation in clutter. In: International Conference on Robotics and Automation. IEEE, pp. 6232-6238.

Newell A, Huang Z and Deng J (2017) Associative embedding: End-to-end learning for joint detection and grouping. In: Advances in Neural Information Processing Systems. pp. 22772287.

Newell A, Yang K and Deng J (2016) Stacked hourglass networks for human pose estimation. In: European Conference on Computer Vision. Springer, pp. 483-499.

Ni P, Zhang W, Zhu X and Cao Q (2020) PointNet++ grasping: Learning an end-to-end spatial grasp generation algorithm from sparse point clouds. In: IEEE International Conference on Robotics and Automation.

Paszke A, Gross S, Chintala S, Chanan G, Yang E, DeVito Z, Lin Z, Desmaison A, Antiga L and Lerer A (2017) Automatic differentiation in PyTorch. In: Neural Information Processing Systems: Autodiff Workshop.

Pelossof R, Miller A, Allen P and Jebara T (2004) An svm learning approach to robotic grasping. In: IEEE International Conference on Robotics and Automation. pp. 3512-3518.

Piater JH (2002) Learning visual features to predict hand orientations. Computer Science Department Faculty Publication Series 148, Univ. of Massachusetts Amherst.

Pinto L and Gupta A (2016) Supersizing self-supervision: Learning to grasp from $50 \mathrm{k}$ tries and 700 robot hours. In: IEEE International Conference on Robotics and Automation. pp. 3406-3413.

Qin Y, Chen R, Zhu H, Song M, Xu J and Su H (2020) S4g: Amodal single-view single-shot se (3) grasp detection in cluttered scenes. In: Conference on Robot Learning. PMLR, pp. 53-65.

Rao D, Le QV, Phoka T, Quigley M, Sudsang A and Ng AY (2010) Grasping novel objects with depth segmentation. In: IEEE/RSJ International Conference on Intelligent Robots and Systems. pp. 2578-2585.
Redmon J and Angelova A (2015) Real-time grasp detection using convolutional neural networks. In: IEEE International Conference on Robotics and Automation. pp. 1316-1322.

Ren S, He K, Girshick R and Sun J (2015) Faster R-CNN: Towards real-time object detection with region proposal networks. In: Advances in Neural Information Processing Systems. pp. 9199.

Sahbani A, El-Khoury S and Bidaud P (2012) An overview of 3D object grasp synthesis algorithms. Robotics and Autonomous Systems 60(3): 326-336.

Satish V, Mahler J and Goldberg K (2019) On-policy dataset synthesis for learning robot grasping policies using fully convolutional deep networks. IEEE Robotics and Automation Letters 4(2): 1357-1364.

Saxena A, Driemeyer J and Ng AY (2008a) Robotic grasping of novel objects using vision. The International Journal of Robotics Research 27(2): 157-173.

Saxena A, Wong LL and Ng AY (2008b) Learning grasp strategies with partial shape information. In: Proceedings of the AAAI Conference on Artificial Intelligence. pp. 1491-1494.

Shao Q, Hu J, Wang W, Fang Y, Liu W, Qi J and Ma J (2019) Suction grasp region prediction using self-supervised learning for object picking in dense clutter. In: IEEE International Conference on Mechatronics System and Robots. pp. 7-12.

Shimoga KB (1996) Robot grasp synthesis algorithms: A survey. The International Journal of Robotics Research 15(3): 230266.

Simonyan K and Zisserman A (2015) Very deep convolutional networks for large-scale image recognition. In: Bengio $\mathrm{Y}$ and LeCun Y (eds.) International Conference on Learning Representations. URL http://arxiv. org/abs/1409. 1556.

Sorour M, Elgeneidy K, Srinivasan A, Hanheide M and Neumann G (2019) Grasping unknown objects based on gripper workspace spheres. In: International Conference on Intelligent Robots and Systems. IEEE, pp. 1541-1547.

Sundermeyer M, Mousavian A, Triebel R and Fox D (2021) Contact-graspnet: Efficient 6-dof grasp generation in cluttered scenes. arXiv preprint arXiv:2103.14127.

ten Pas A, Gualtieri M, Saenko K and Platt R (2017) Grasp pose detection in point clouds. The International Journal of Robotics Research 36(13-14): 1455-1473.

ten Pas A and Platt R (2018) Using geometry to detect grasp poses in 3D point clouds. In: Robotics Research. Springer, pp. 307324.

Tremblay J, To T, Sundaralingam B, Xiang Y, Fox D and Birchfield S (2018) Deep object pose estimation for semantic robotic grasping of household objects. In: Conference on Robot Learning. pp. 306-316.

Varley J, Weisz J, Weiss J and Allen P (2015) Generating multifingered robotic grasps via deep learning. In: IEEE/RSJ International Conference on Intelligent Robots and Systems. pp. 4415-4420.

Veres M, Moussa M and Taylor GW (2017) Modeling grasp motor imagery through deep conditional generative models. IEEE Robotics and Automation Letters 2(2): 757-764.

Viereck U, Pas At, Saenko K and Platt R (2017) Learning a visuomotor controller for real world robotic grasping using simulated depth images. In: Conference on Robot Learning. pp. 291-300. 
Wang X, Girshick R, Gupta A and He K (2018) Non-local neural networks. In: IEEE Conference on Computer Vision and Pattern Recognition. pp. 7794-7803.

Wang Z, Li Z, Wang B and Liu H (2016) Robot grasp detection using multimodal deep convolutional neural networks. Advances in Mechanical Engineering 8(9): 1687814016668077.

Watson J, Hughes J and Iida F (2017) Real-world, real-time robotic grasping with convolutional neural networks. In: Annual Conference Towards Autonomous Robotic Systems. Springer, pp. 617-626.

Wu C, Chen J, Cao Q, Zhang J, Tai Y, Sun L and Jia K (2020) Grasp proposal networks: An end-to-end solution for visual learning of robotic grasps. arXiv preprint arXiv:2009.12606 .

Xu R, Chu FJ, Tang C, Liu W and Vela PA (2021) An affordance keypoint detection network for robot manipulation. IEEE Robotics and Automation Letters 6(2): 2870-2877.

$\mathrm{Xu} \mathrm{R}$, Chu FJ and Vela PA (2020a) The abridged Jacquard dataset. https://smartech.gatech.edu/handle/ $1853 / 64897$.

Xu R, Chu FJ and Vela PA (2020b) IVALAb: Grasp Keypoint Network git repository. https://github.com/ivalab/ GraspKpNet.

Yen-Chen L, Zeng A, Song S, Isola P and Lin TY (2020) Learning to see before learning to act: Visual pre-training for manipulation. In: IEEE International Conference on Robotics and Automation.

Yu F, Wang D, Shelhamer E and Darrell T (2018) Deep layer aggregation. In: IEEE Conference on Computer Vision and Pattern Recognition. pp. 2403-2412.

Zapata-Impata BS, Mateo Agulló C, Gil P and Pomares J (2017) Using geometry to detect grasping points on $3 \mathrm{~d}$ unknown point cloud .

Zeng A, Song S, Welker S, Lee J, Rodriguez A and Funkhouser T (2018a) Learning synergies between pushing and grasping with self-supervised deep reinforcement learning. In: IEEE/RSJ International Conference on Intelligent Robots and Systems. pp. 4238-4245.

Zeng A, Song S, Yu KT, Donlon E, Hogan FR, Bauza M, Ma D, Taylor O, Liu M, Romo E, Nima F, Ferran A, Dafle NC, Holladay R, Morona I, Nair PQ, Green D, Taylor I, Liu W, Funkhouser T and Alberto R (2018b) Robotic pick-andplace of novel objects in clutter with multi-affordance grasping and cross-domain image matching. In: IEEE International Conference on Robotics and Automation. pp. 1-8.

Zhao B, Zhang H, Lan X, Wang H, Tian Z and Zheng N (2020) Regnet: region-based grasp network for single-shot grasp detection in point clouds. arXiv preprint arXiv:2002.12647 .

Zhou X, Lan X, Zhang H, Tian Z, Zhang Y and Zheng N (2018) Fully convolutional grasp detection network with oriented anchor box. In: IEEE/RSJ International Conference on Intelligent Robots and Systems. pp. 7223-7230.

Zhou Y and Hauser K (2017) 6DOF grasp planning by optimizing a deep learning scoring function. In: Robotics: Science and Systems Workshop on Revisiting Contact-Turning a Problem into a Solution. 